\title{
Experiments in nuclear astrophysics II (neutron-induced)
}

\author{
René Reifarth
}

GSI Darmstadt/University of Frankfurt

WE-Heraeus Summer School on Nuclear Astrophysics in the Cosmos Darmstadt/Heidelberg 12-17 July 2010 


\section{solar abundance distribution}

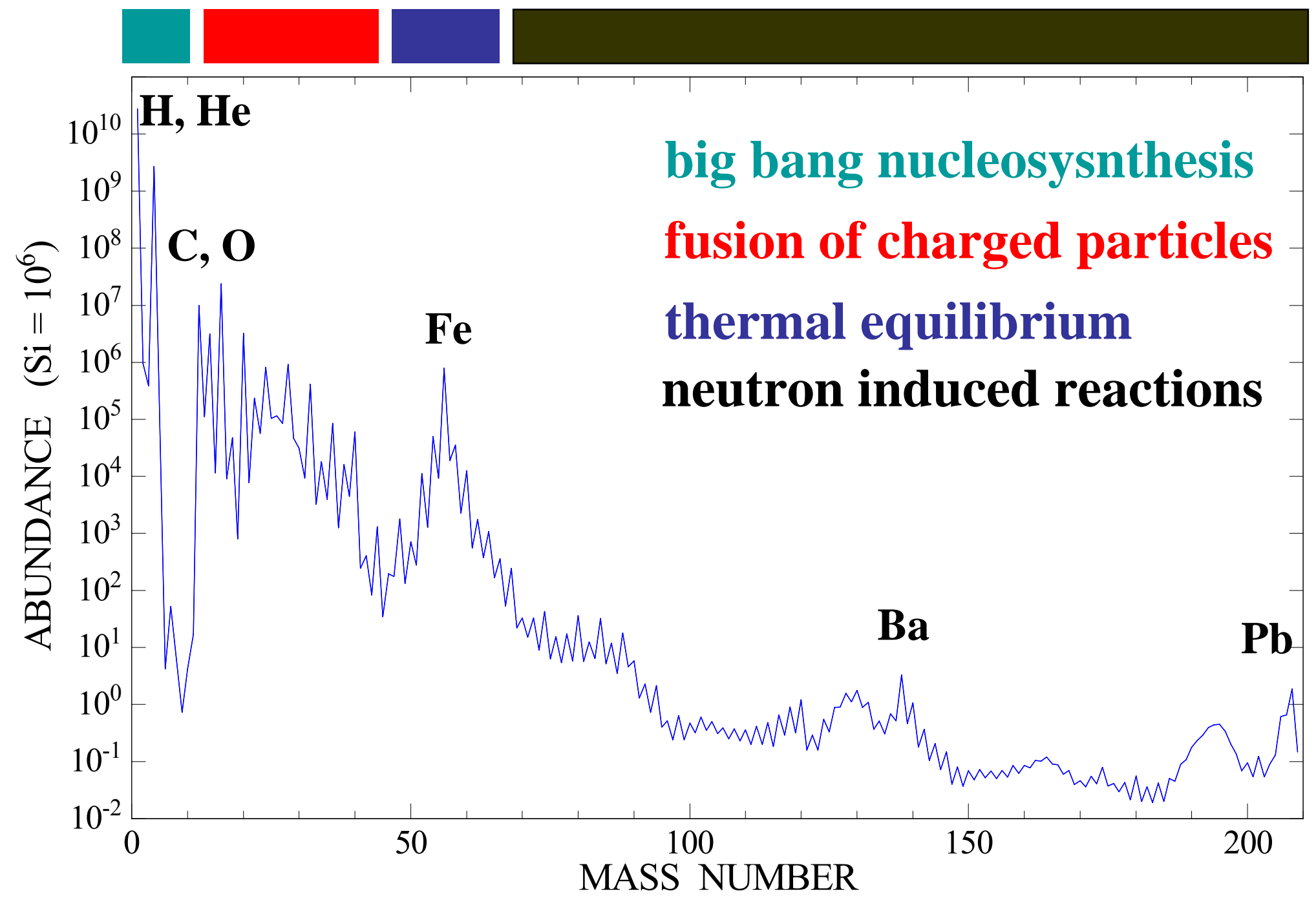

René Reifarth (GSI / U. Frankfurt) 


\section{Nucleosynthesis of the elements}

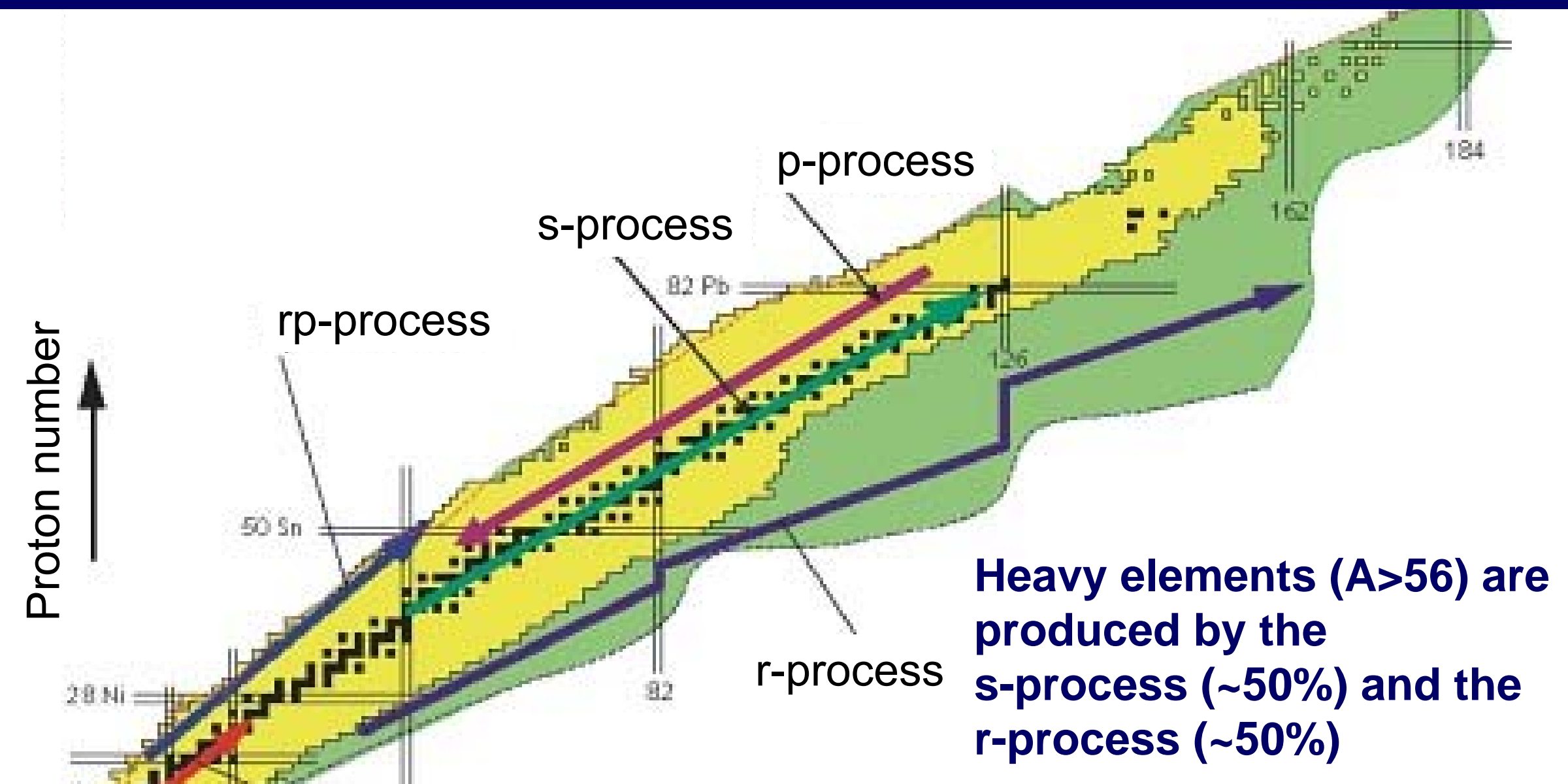

Neutron number

René Reifarth (GSI / U. Frankfurt) 


\section{the s-process}

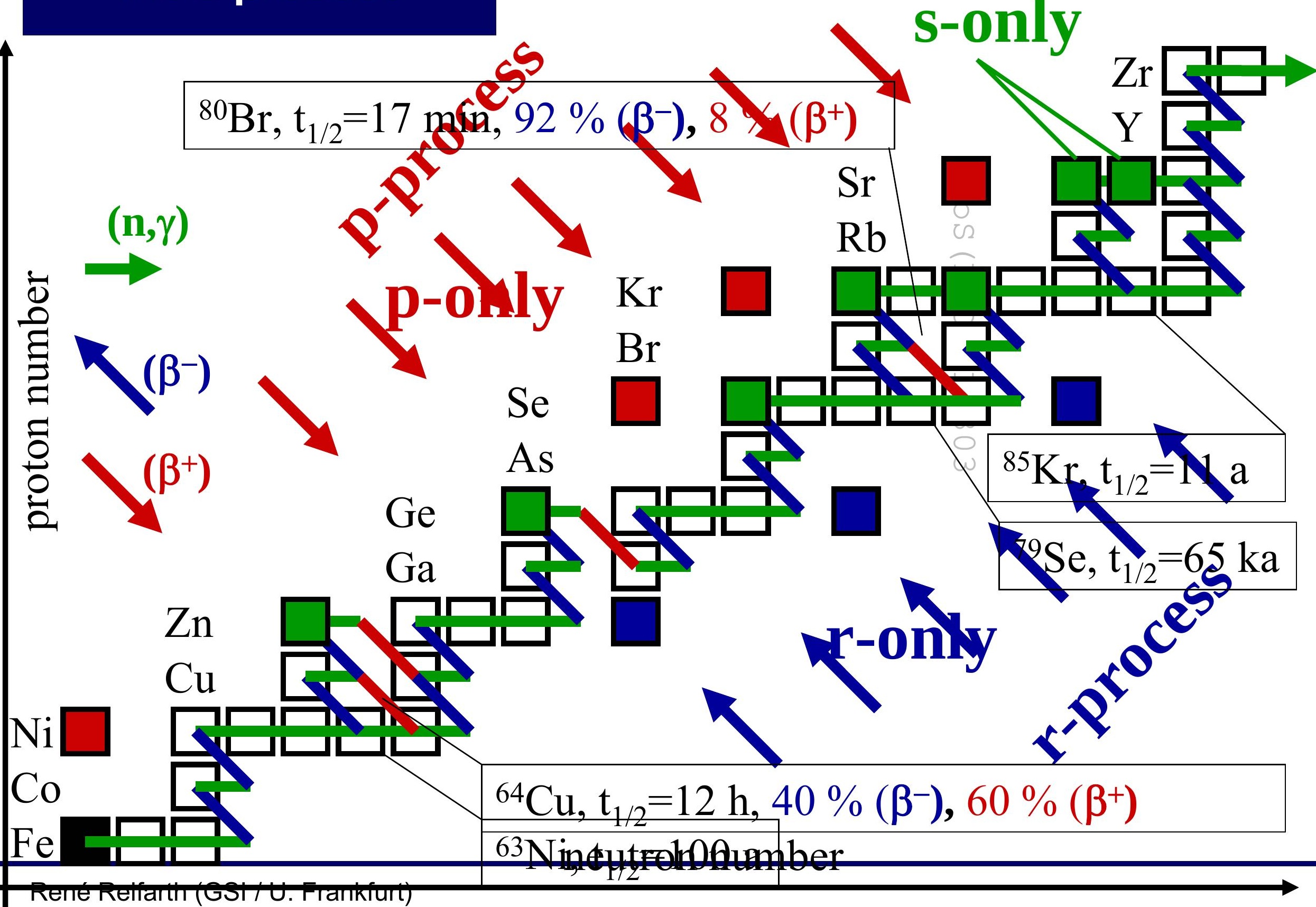




\section{- Reaction rates}

- Neutron induced (1-200 keV)

- Charged particles

- Half-lives Neutrons

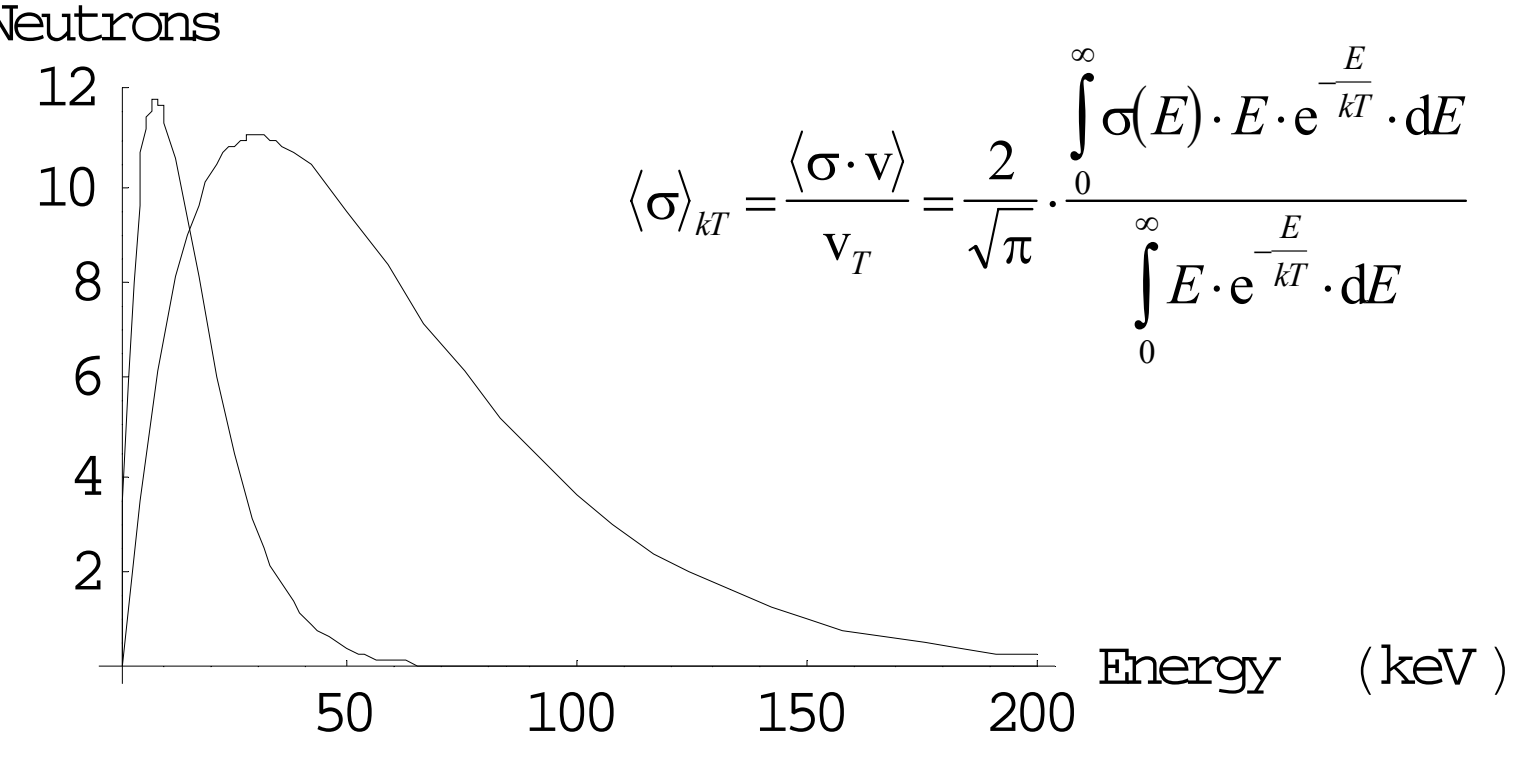


Stellar model vs. experiment

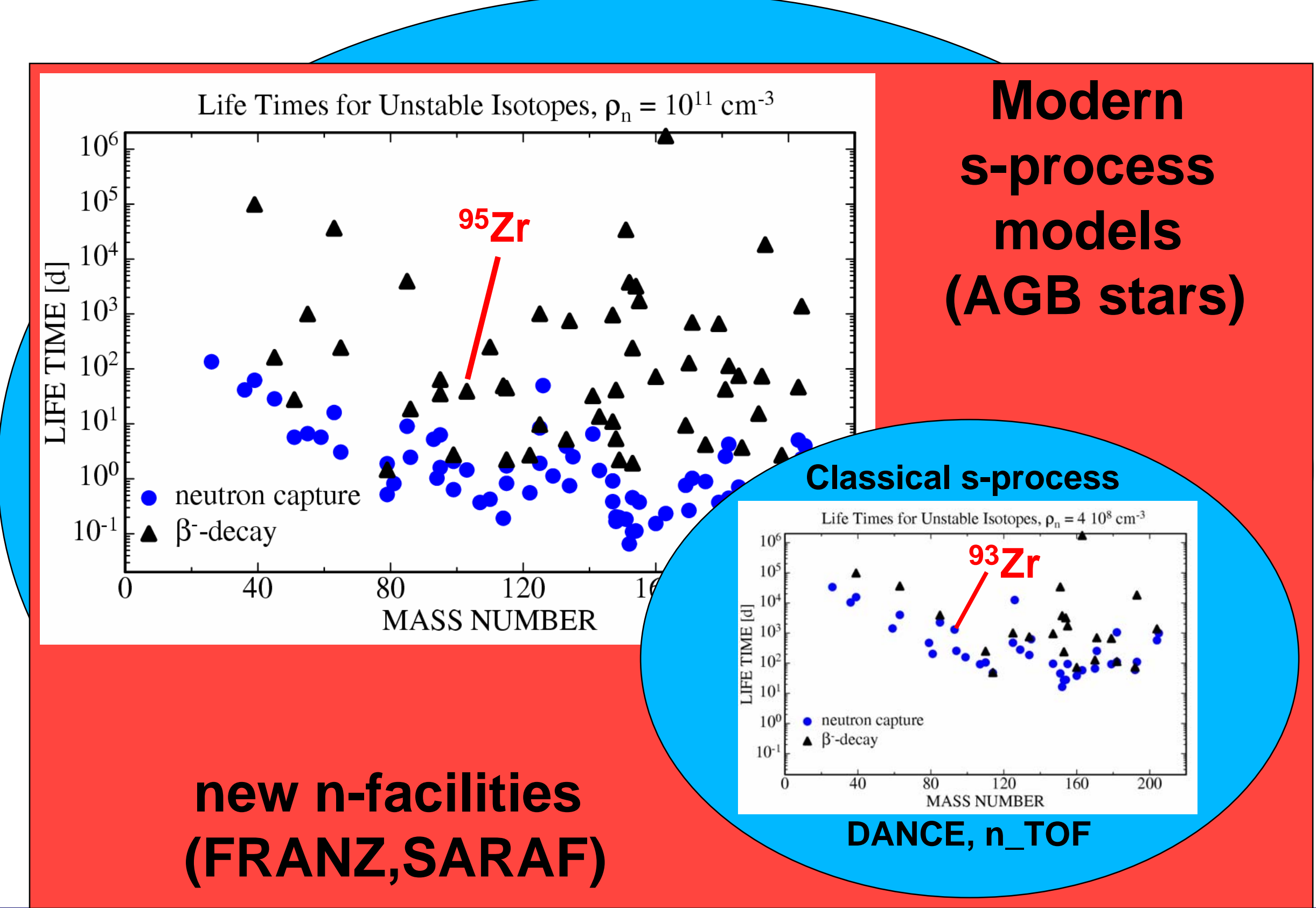

René Reifarth (GSI / U. Frankfurt) 


\section{Neutron Captures - time-of-flight technique}

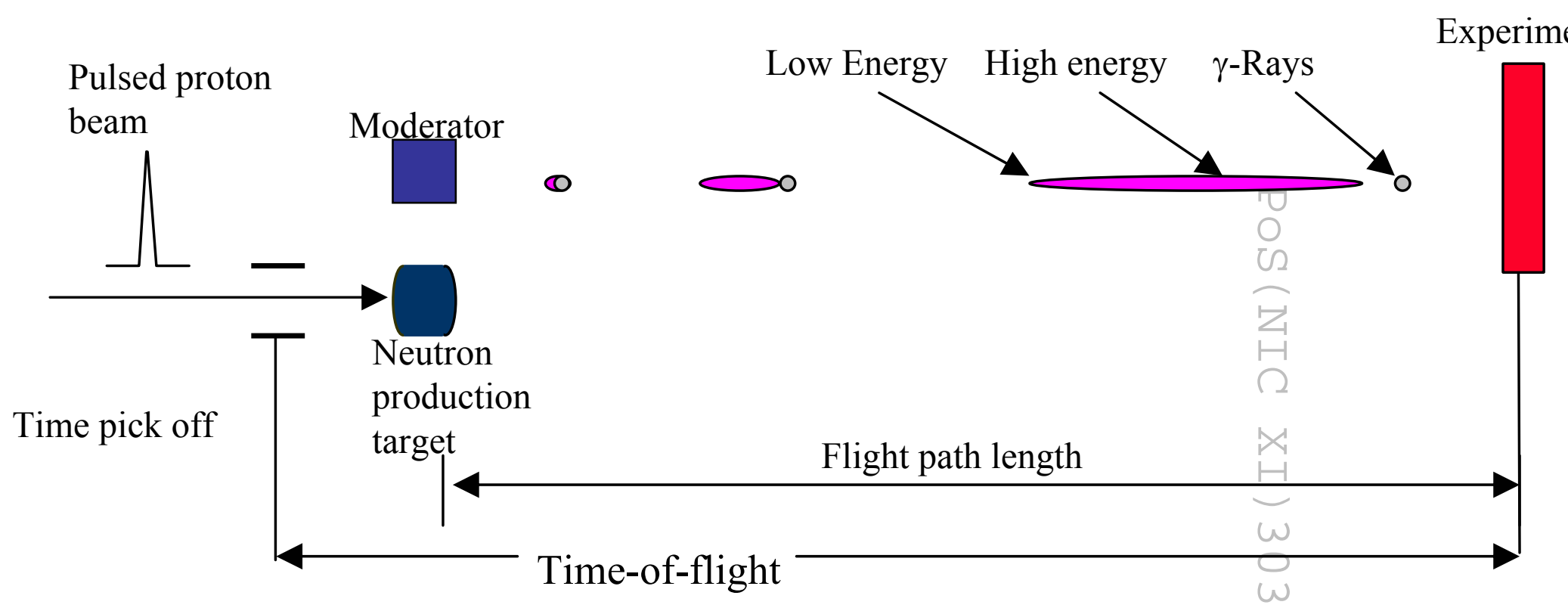

Example:
$\mathrm{L}=20 \mathrm{~m}$

TOF $_{\mathrm{g}}=67 \mathrm{~ns}$

$\Delta$ TOF $=5 \mathrm{~ns}$ $\Delta$ TOF $=0.7 \mu \mathrm{s}$ $\triangle T O F=1 \mathrm{~ms}$

\begin{tabular}{|c|c|c|c|}
\hline \multicolumn{4}{|c|}{ Astrophysics } \\
\hline$E_{n}=1 \mathrm{MeV}$ & $E_{n}=100 \mathrm{keV}$ & $E_{n}=10 \mathrm{keV}$ & $E_{n}=1 \mathrm{eV}$ \\
\hline $\mathrm{TOF}_{\mathrm{n}}=1.5 \mu \mathrm{s}$ & $\mathrm{TOF}_{\mathrm{n}}=4.6 \mu \mathrm{s}$ & $\mathrm{TOF}_{\mathrm{n}}=15 \mu \mathrm{s}$ & $\mathrm{TOF}_{\mathrm{n}}=1.5 \mathrm{~ms}$ \\
\hline $\begin{array}{l}\Delta E=7 \mathrm{keV} \\
\Delta E=1.6 \mathrm{Me} \\
E>2 \mathrm{eV}\end{array}$ & $\begin{array}{l}\Delta E=0.2 \mathrm{keV} \\
\Delta E=32 \mathrm{keV} \\
E>2 \mathrm{eV}\end{array}$ & $\begin{array}{l}\Delta E=7 \mathrm{eV} \\
\Delta E=1 \mathrm{keV} \\
E>2 \mathrm{eV}\end{array}$ & $\begin{array}{l}\Delta \mathrm{E}=0.01 \mathrm{meV} \\
\Delta \mathrm{E}=1 \mathrm{meV} \\
\Delta \mathrm{E}=5 \mathrm{eV}\end{array}$ \\
\hline
\end{tabular}

Astrophysics

$E_{\mathrm{n}}=100 \mathrm{keV} \quad E_{\mathrm{n}}=10 \mathrm{keV} \quad E_{\mathrm{n}}=1 \mathrm{eV}$

$\mathrm{TOF}_{\mathrm{n}}=1.5 \mathrm{~ms}$

René Reifarth (GSI / U. Frankfurt) 


\section{branch point in the s-process path}

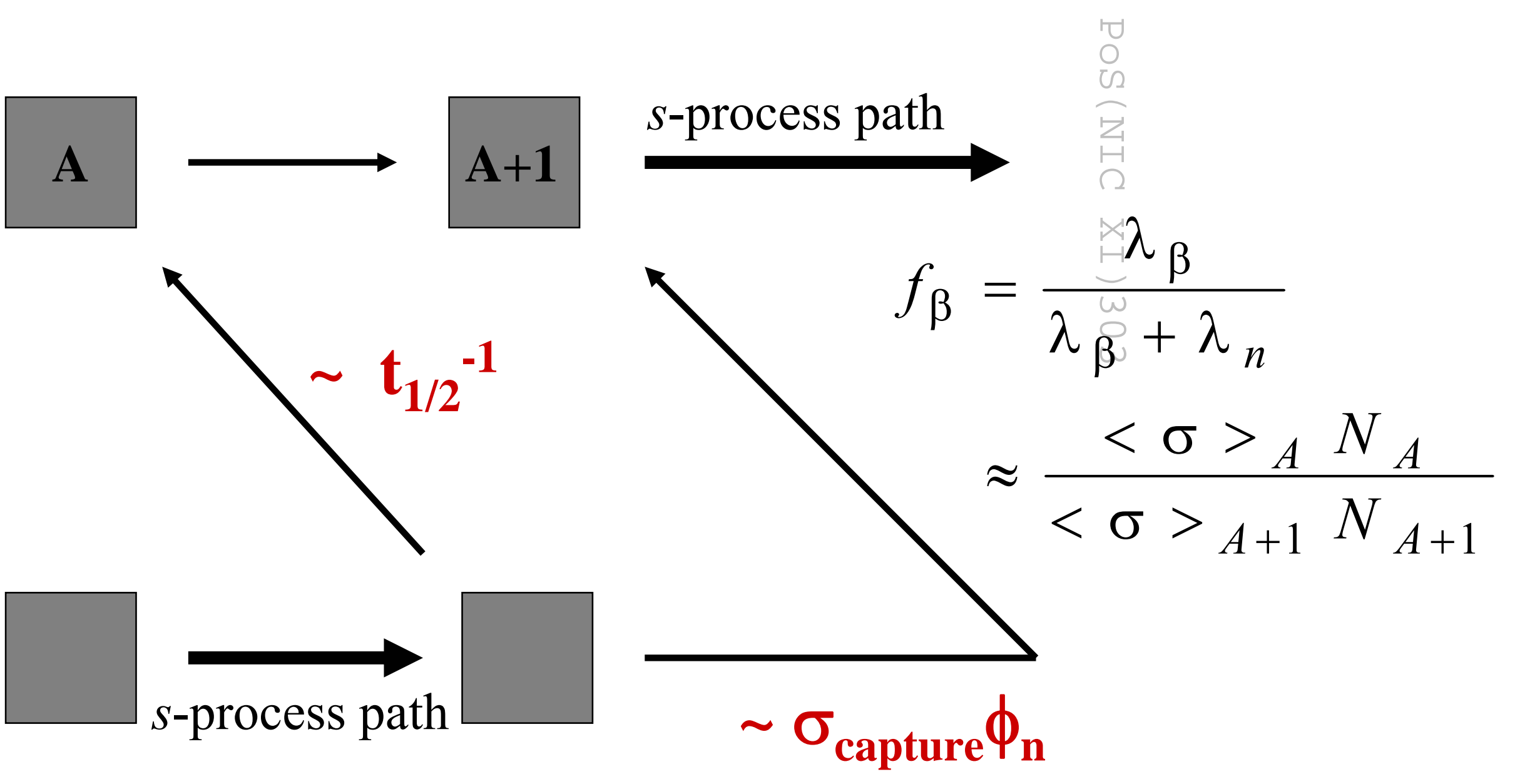




\section{$(n, g)$ experiments with unstable isotopes and fundamental stellar physics evaluations}

\begin{tabular}{|c|c|c|c|c|c|}
\hline $\begin{array}{l}\text { Branch } \\
\text { Isotope }\end{array}$ & $\begin{array}{l}\text { Half- } \\
\text { Life }\end{array}$ & Facility & Observable & $\begin{array}{c}\text { Stellar Physics } \\
10 \\
0 \\
\ddots 2 \\
\end{array}$ & Comment \\
\hline${ }^{151} \mathrm{Sm}$ & $93 \mathrm{yr}$ & $\begin{array}{l}\text { FZK, } \\
\text { n_TOF, } \\
\text { DANCE }\end{array}$ & $\begin{array}{l}{ }^{152} \mathrm{Gd} \text { in solar } \\
\text { distribution } \\
{ }^{151} \mathrm{Eu} / 153 \mathrm{Eu} \text { ratio } \\
\text { hyperfine line } \\
\text { split }\end{array}$ & $\begin{array}{l}\text { Timescale of hot } \\
\text { Helium-shell } \\
\text { flash } \\
\text { s-processin very } \\
\text { old stars }\end{array}$ & done \\
\hline${ }^{134} \mathrm{Cs}$ & $2 \mathrm{yr}$ & $\begin{array}{l}\text { DANCE, } \\
\text { FRANZ }\end{array}$ & $\begin{array}{l}\text { Ba isotope ratios } \\
\text { from presolar } \\
\text { grains }\end{array}$ & $\begin{array}{l}\text { Sets }{ }^{12} \mathrm{C} \\
\text { abundance } \\
\text { of He-shell flash }\end{array}$ & $\begin{array}{l}\text { current } \\
\text { uncertainty: } \\
\pm 30 \%\end{array}$ \\
\hline${ }^{135} \mathrm{Cs}$ & $2 \mathrm{Myr}$ & & $\mathrm{Ba}$ isotope ratios & $\begin{array}{l}\text { Amount of } \\
\text { rotation }\end{array}$ & $\pm 10 \%$ \\
\hline${ }^{95} \mathrm{Zr}$ & $64 \mathrm{~d}$ & $\begin{array}{l}\text { FRANZI } \\
\text { FAIR }\end{array}$ & $\begin{array}{l}{ }^{96} / \mathrm{Zr} /{ }^{94} \mathrm{Zr} \text { ratio } \\
\text { presolar grains }\end{array}$ & $\begin{array}{l}\text { Temperature at } \\
\text { bottom of He- } \\
\text { shell flash region }\end{array}$ & $\begin{array}{l}\text { Current } \\
\text { uncertainty: } \\
20-80 \mathrm{mb}\end{array}$ \\
\hline
\end{tabular}




\section{experimental problems}

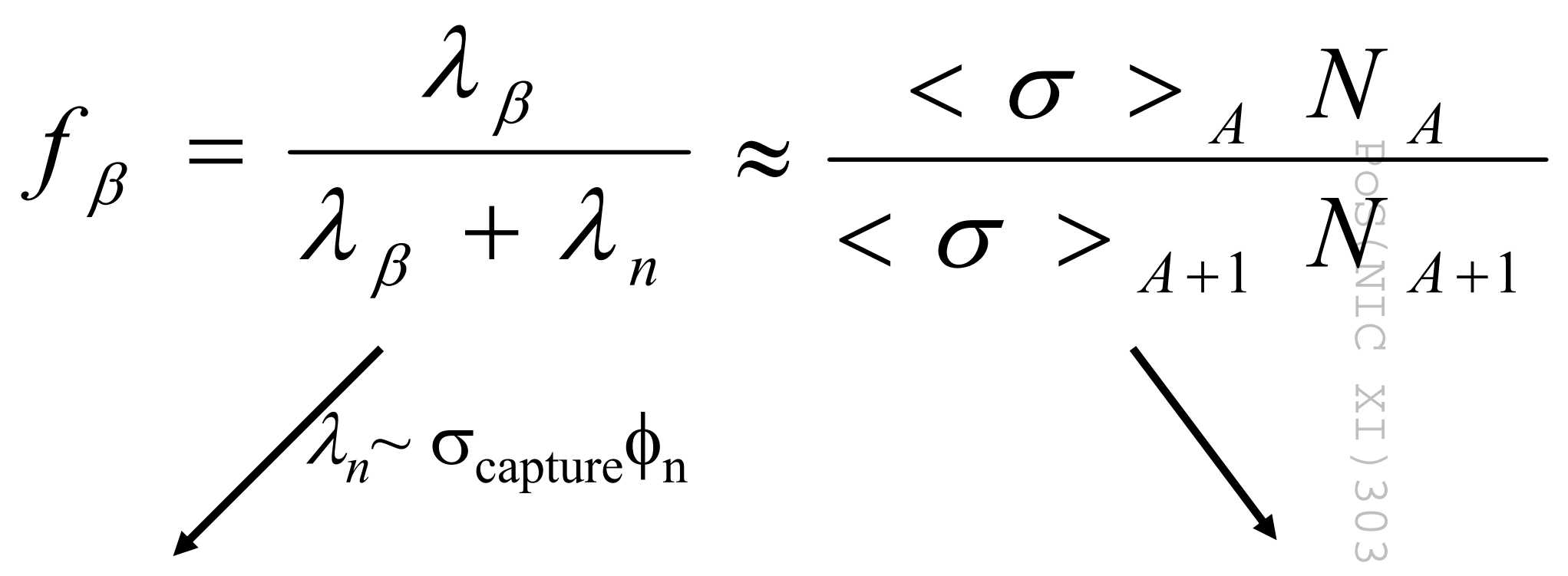

unstable isotope

- sample preparation

- very small amounts

- radioactive background high precision

- neutron induced background

- isotopic impurities

- statistics

\section{$\Rightarrow$ activation technique}

$\Rightarrow$ TOF technique,

$\Rightarrow$ calorimetric 


\section{Red Giants - easy to spot}
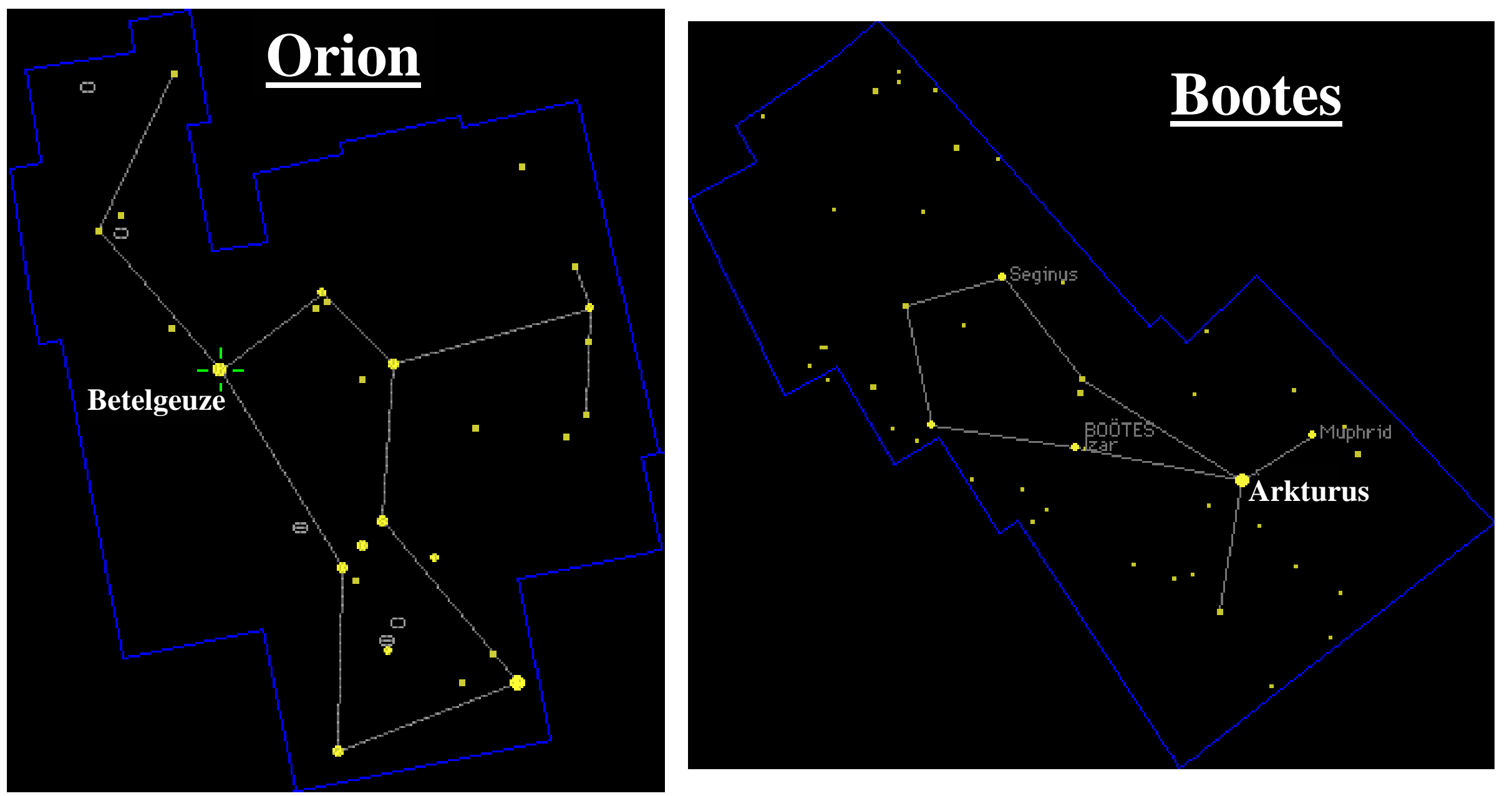

René Reifarth (GSI / U. Frankfurt) 


\section{Red Giants become White Dwarfs}

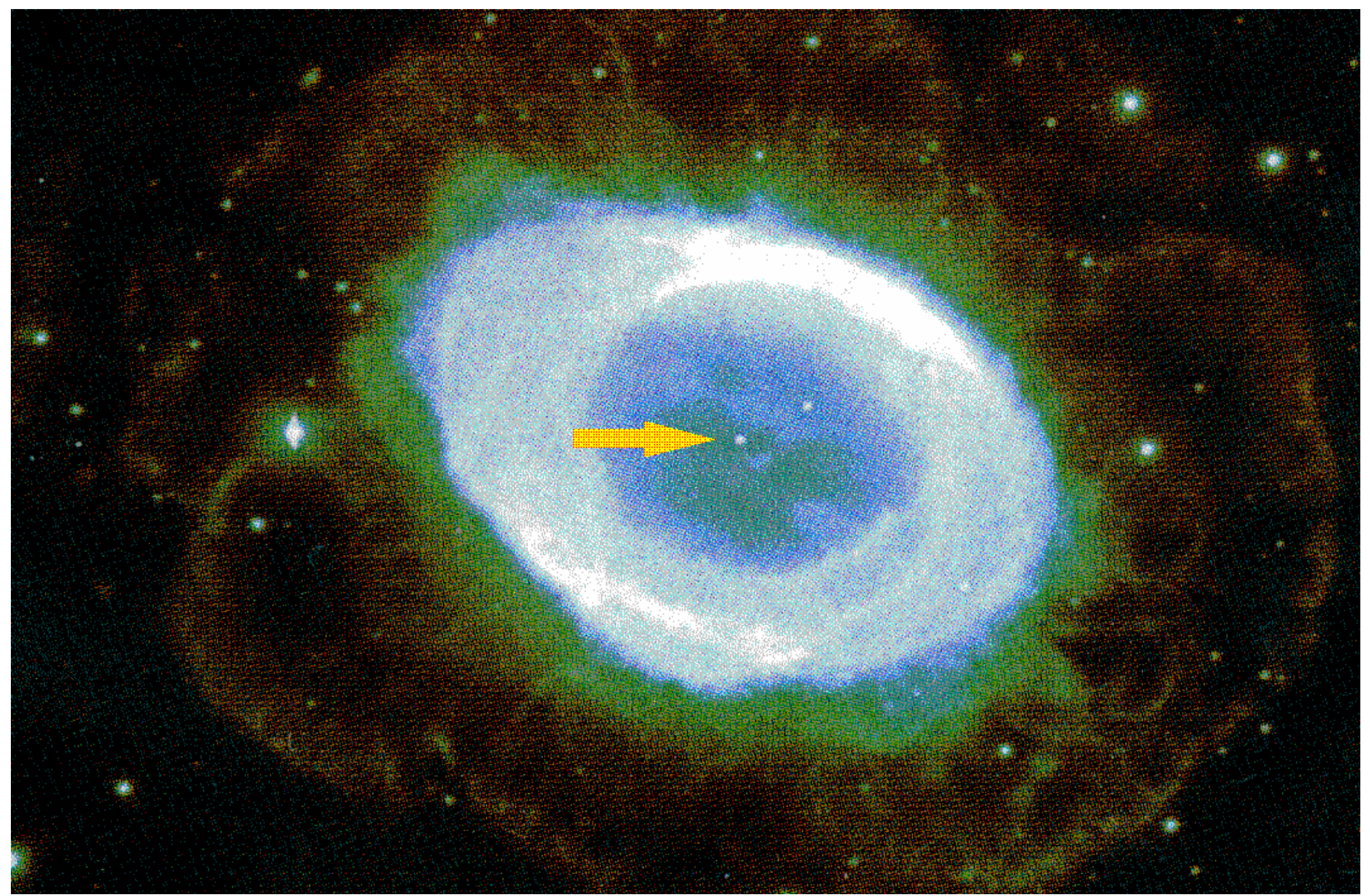

Ring nebula illuminated by the White Dwarf in the center.

\section{René Reifarth (GSI / U. Frankfurt)}




\section{branch point at ${ }^{128}$}

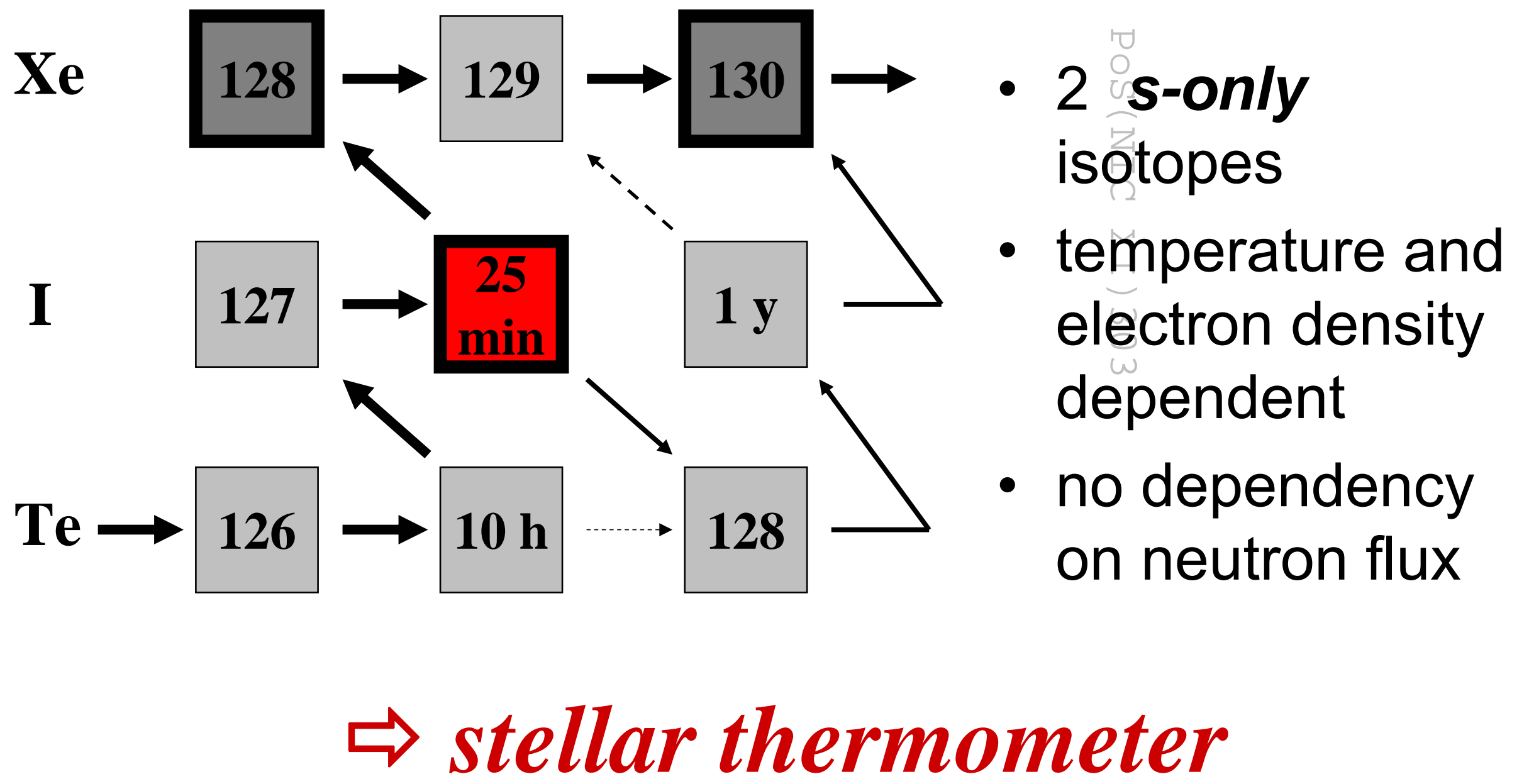




\section{Meteorites - hints from the sky}
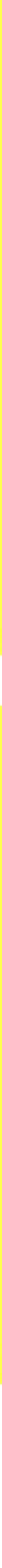

See lecture by Ernst Zinner!

René Reifarth (GSI / U. Frankfurt) 


\section{evidence for neutron capture: DIRECT}

$$
{ }^{A} X+n \Rightarrow{ }^{A+1} X+Q
$$

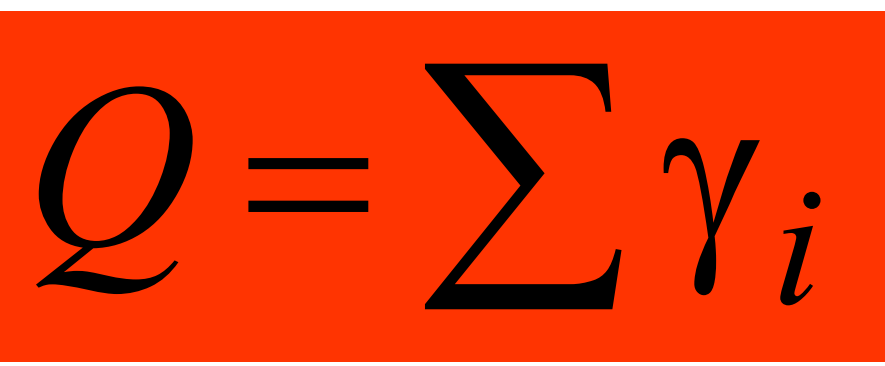

\section{$\Rightarrow$ "monoenergetic" if $100 \%$ efficiency}




\section{The ${ }^{7} \mathrm{Li}(p, n)$ reaction}

- Negative Q-value (-1.644 MeV)

- Neutron spectrum close to threshold depends strongly on proton energy

- Q-value in reach for small accelerators

- Huge cross section close to threshold

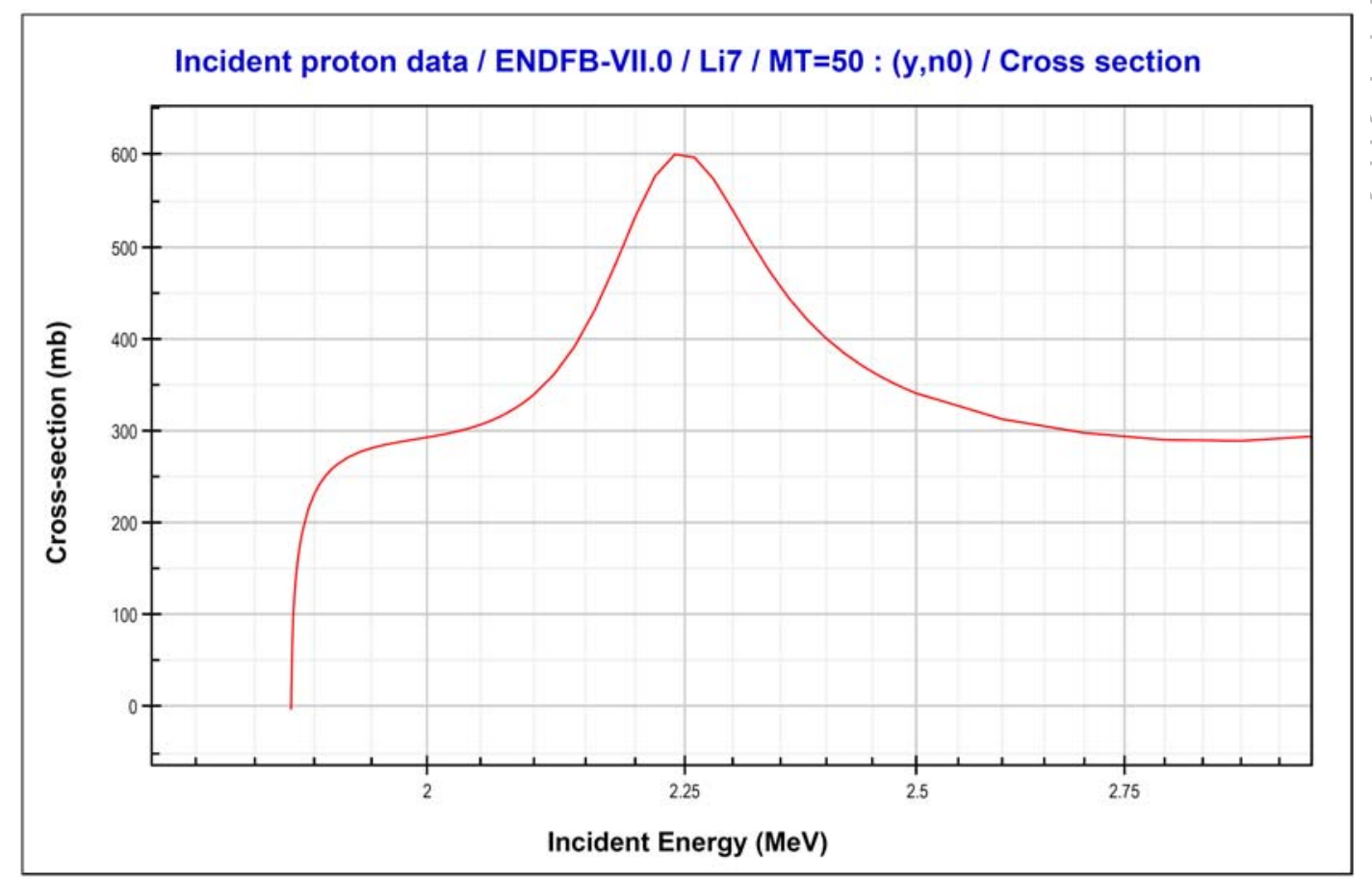




\section{experimental setup}

\section{neutrons:}

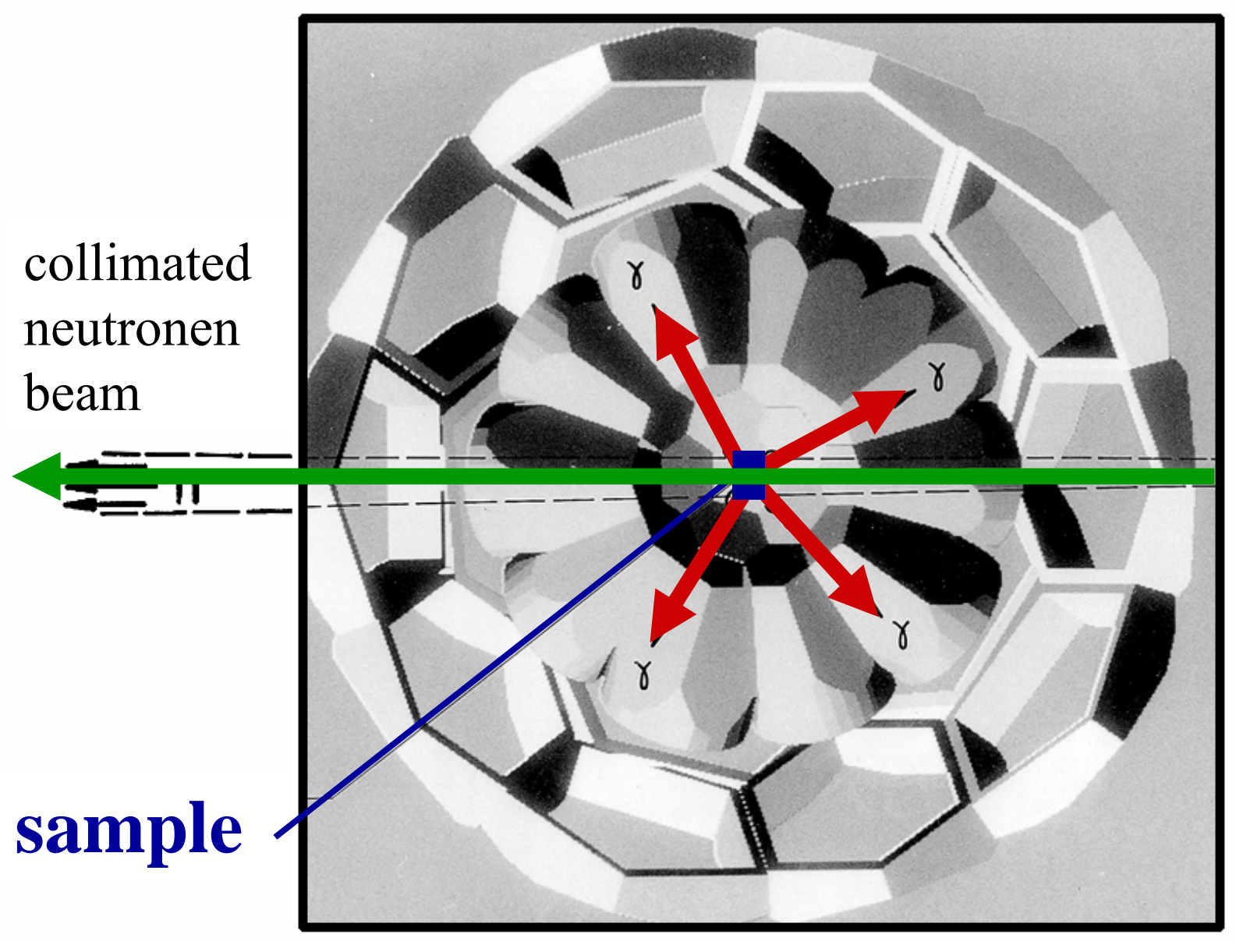

- ${ }^{7} \mathbf{E i}(p, n)$

-1.200 keV

- $10^{4} \mathrm{n} / \mathrm{s} \mathrm{cm}^{2}$

-80 cm flight path

w

$\omega$

$\gamma$-Detector:

- $41 \mathrm{BaF}_{2}$ crystals

- $15 \mathrm{~cm}$ length

- $\varepsilon_{\gamma} \approx 90 \%$

- $\varepsilon_{\text {casc }} \approx 98 \%$

René Reifarth (GSI / U. Frankfurt) 


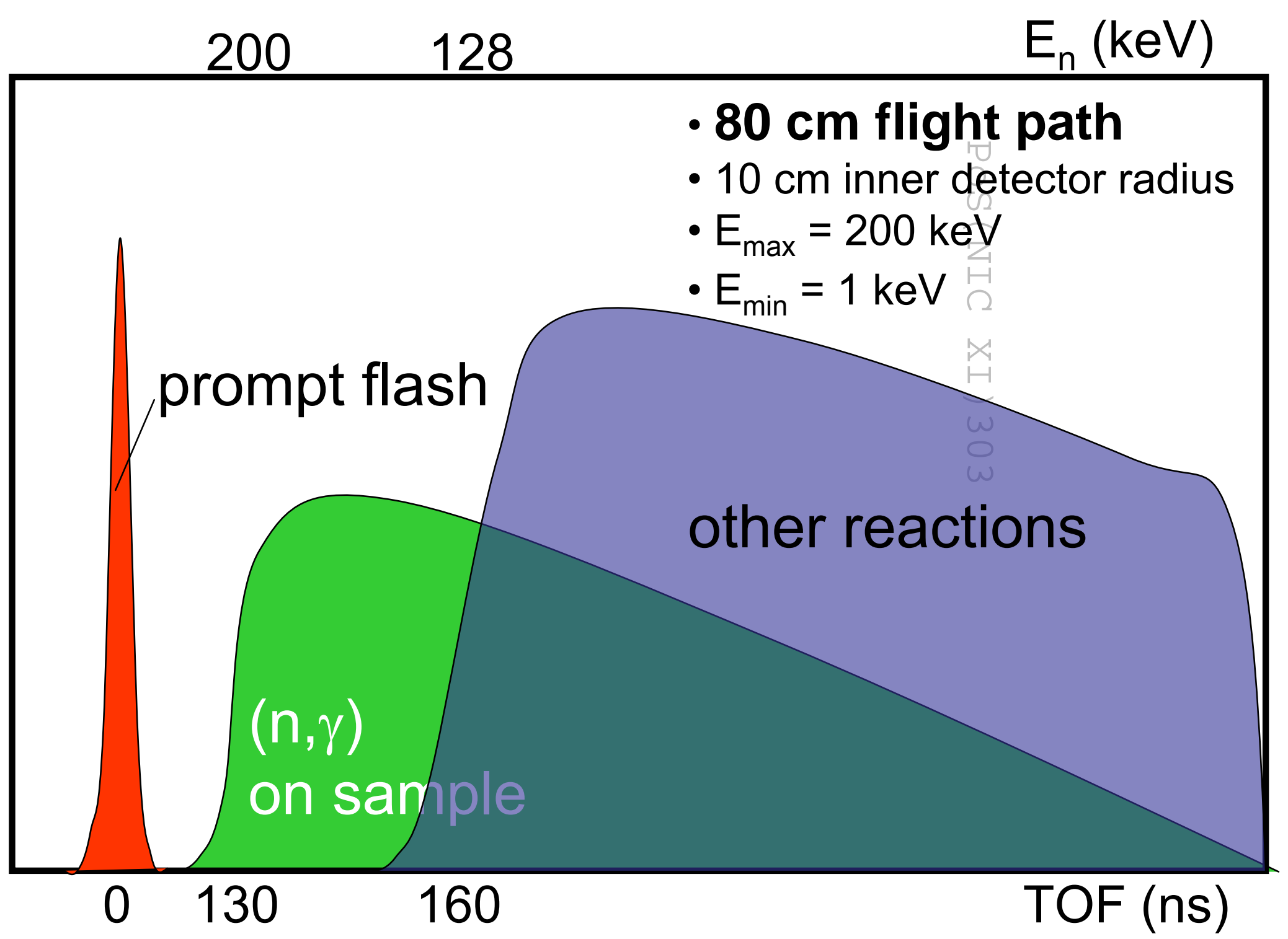

René Reifarth (GSI / U. Frankfurt) 


\section{samples}

- isotopically enriched $128,129,130 \mathrm{Xe}$

- 0.5 .. $1 \mathrm{~g}$ per sample

- filled in Ti-spheres $\left(R_{\text {in }}=4.8 \mathrm{~mm}, R_{\text {out }}=5\right.$ $\mathrm{mm}$ )

- $p=60$ bar 


\section{sum spectra}

- Peak at neutron-binding energy

- $\varepsilon_{\text {casc }}=96 . .98 \%$

- energy threshold: 1.6 $\mathrm{MeV}$

- relative to ${ }^{197} \mathrm{Au}(\mathrm{n}, \gamma)$

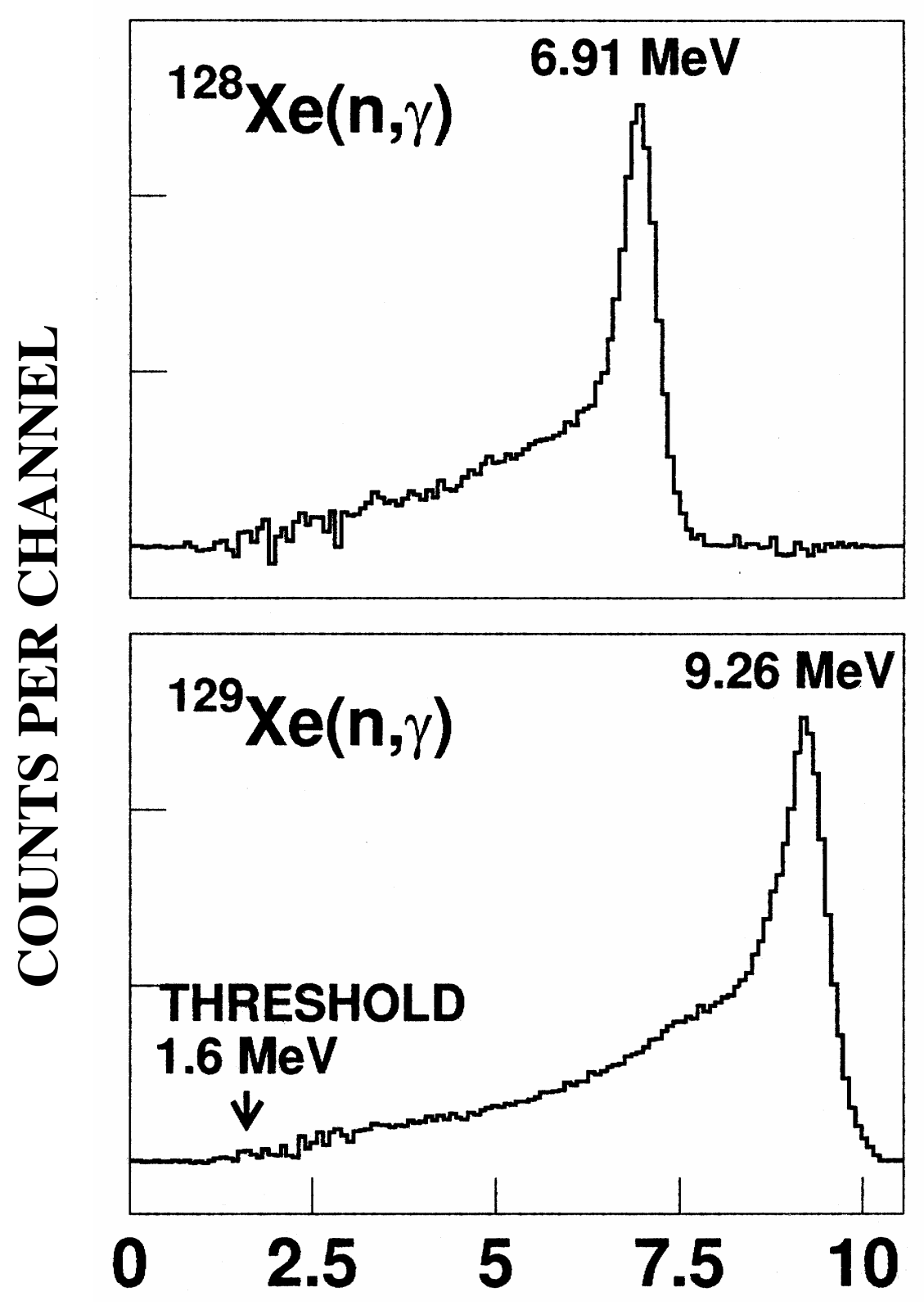

GAMAMA ENEREY (MEV) 


\section{multiplicity}

\section{discrimination of}

natural background

$(\mathrm{n}, \gamma):$

$90 \%$ multiplicity $\geq 3$

natural background:

multiplicity $\leq 2$.
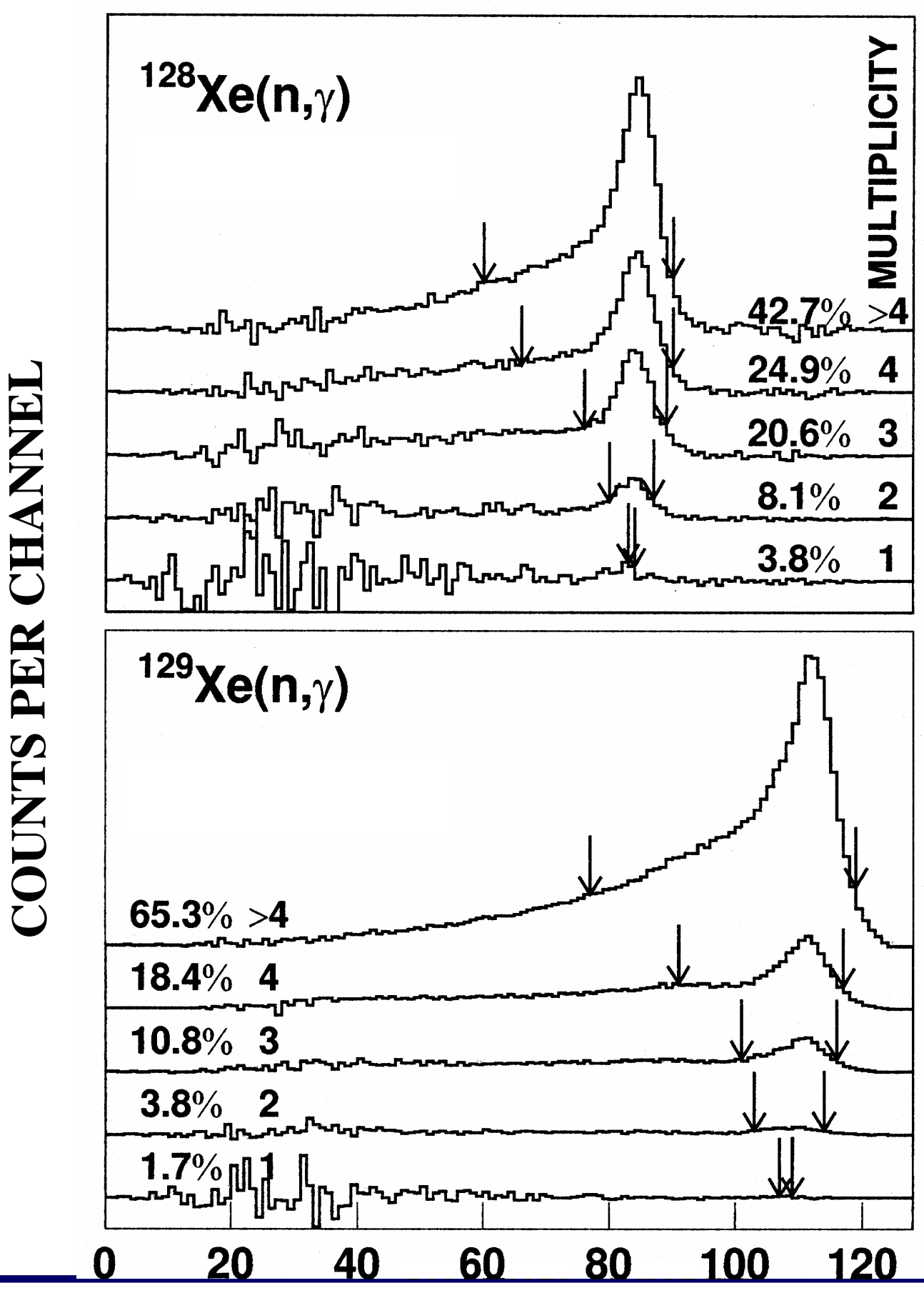


\section{sample dependent background}

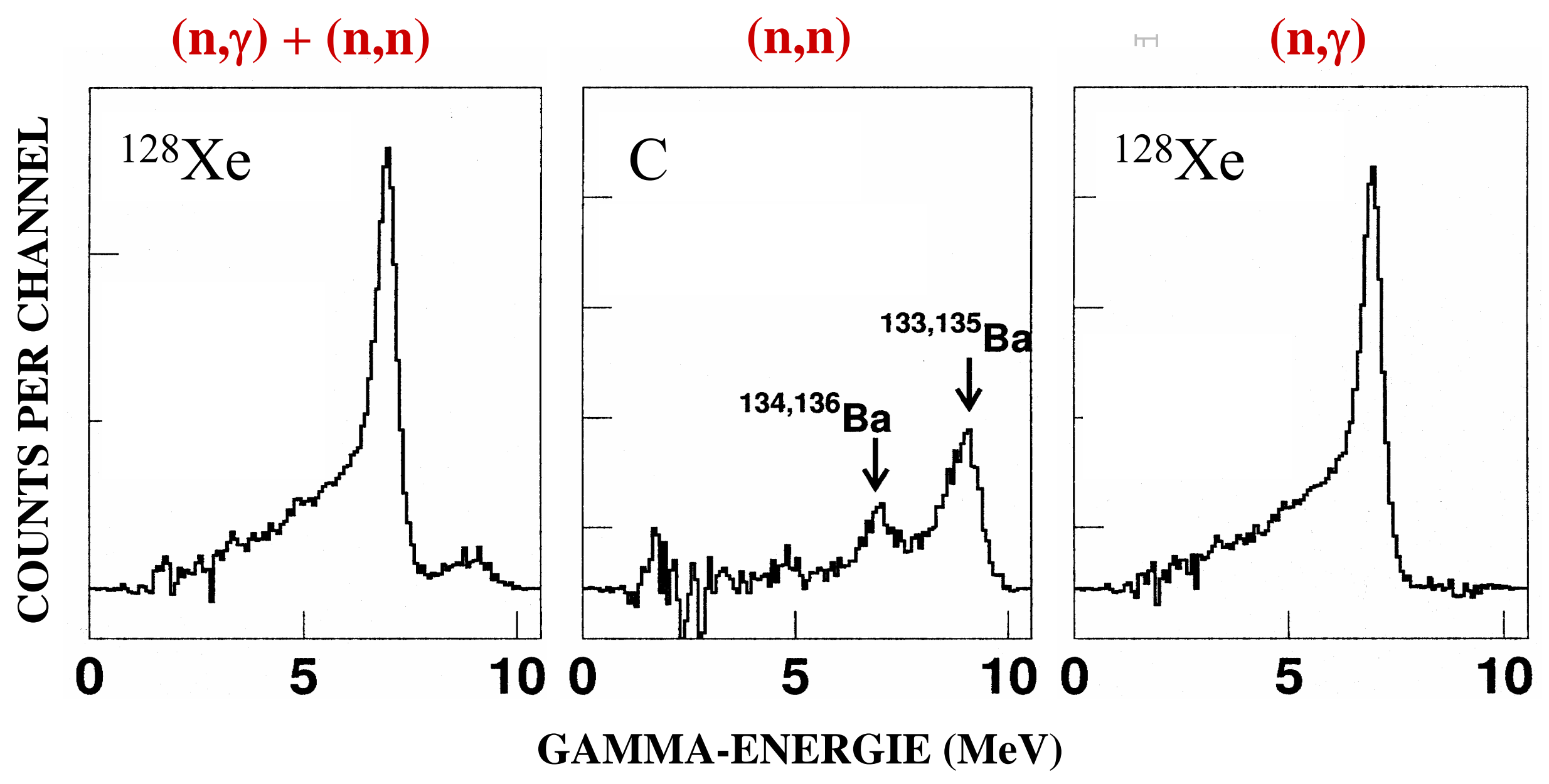

René Reifarth (GSI / U. Frankfurt) 


\section{time of flight}

- background due to scattered neutrons delayed

- $\sigma_{\text {tot }} / \sigma_{\gamma}\left({ }^{128} \mathrm{Xe}\right)=$ 25.

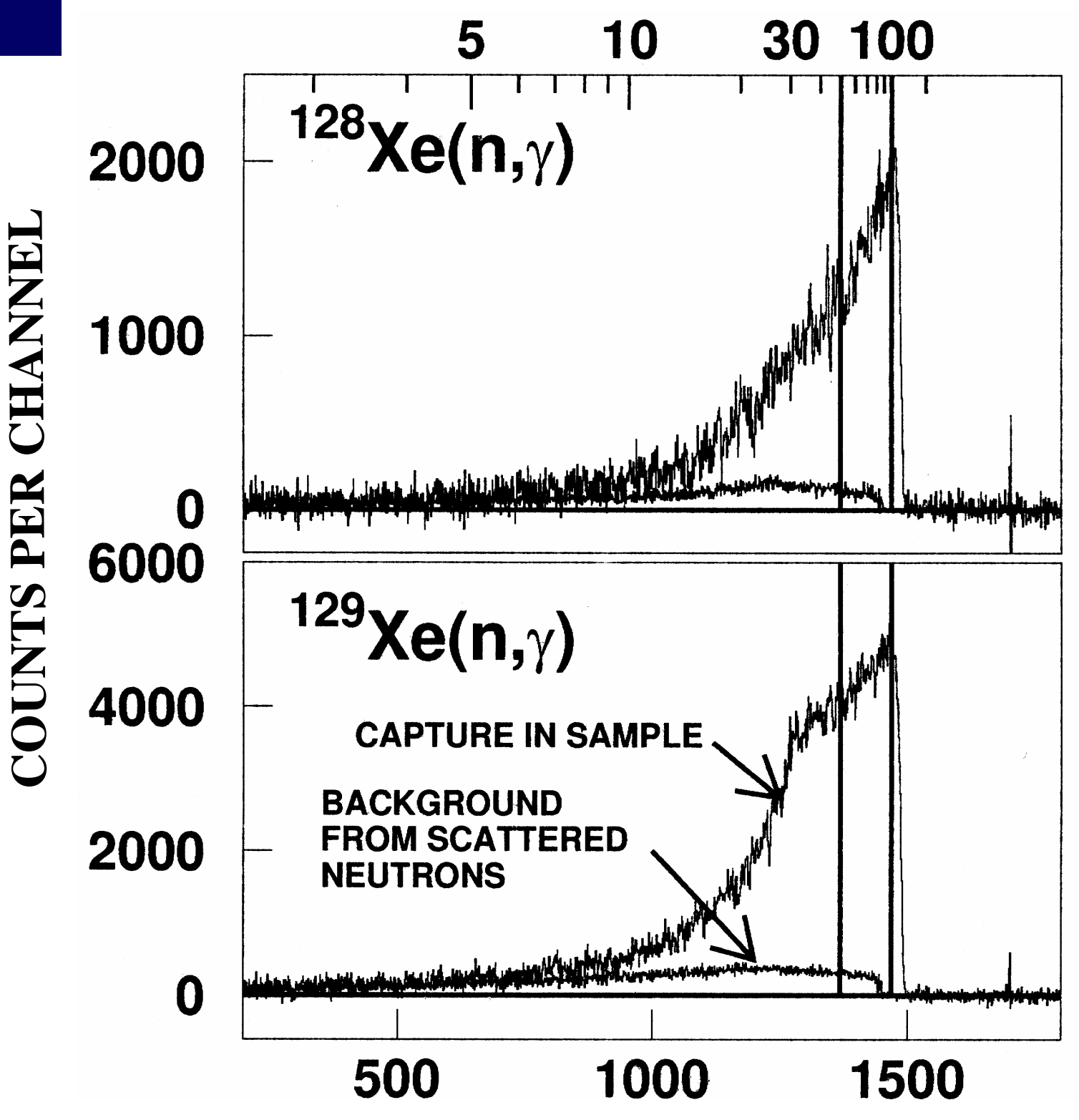




\section{$128,129,130 \mathrm{Xe}(\mathrm{n}, \gamma)$-cross sections}

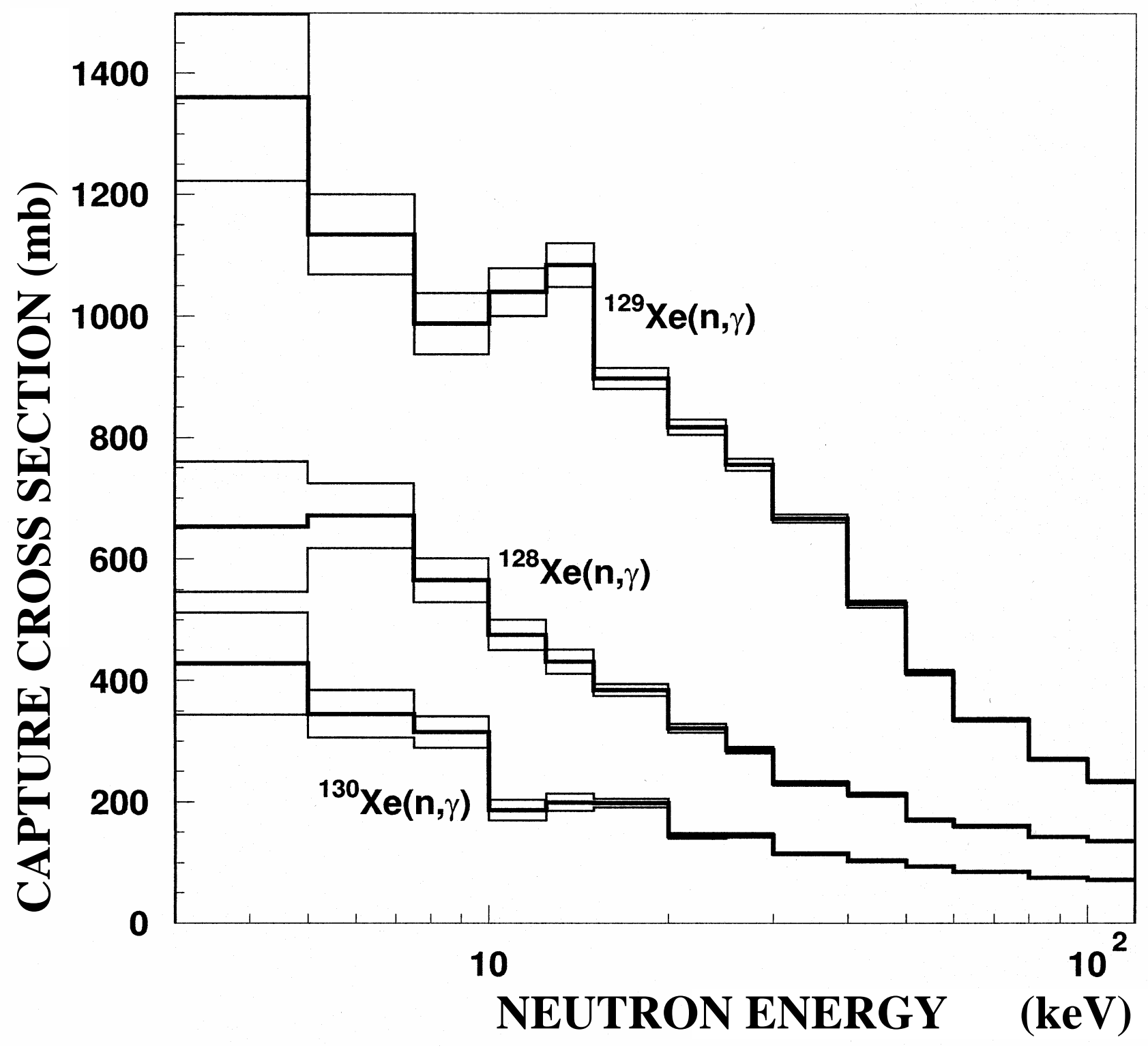

René Reifarth (GSI / U. Frankfurt) 


\section{Spallation neutron sources}

- Need to increase neutrons/proton

- $n / p=10^{-6}$ for ${ }^{7} \operatorname{Li}(p, n)$

- Idea: high energy protons use most of their energy to knock out neutrons from a heavy nucleus - spallation

- Now n/p between 20 (LANSCE,SNS) and 250 (n_TOF) 


\section{LANSCE @ LANL}

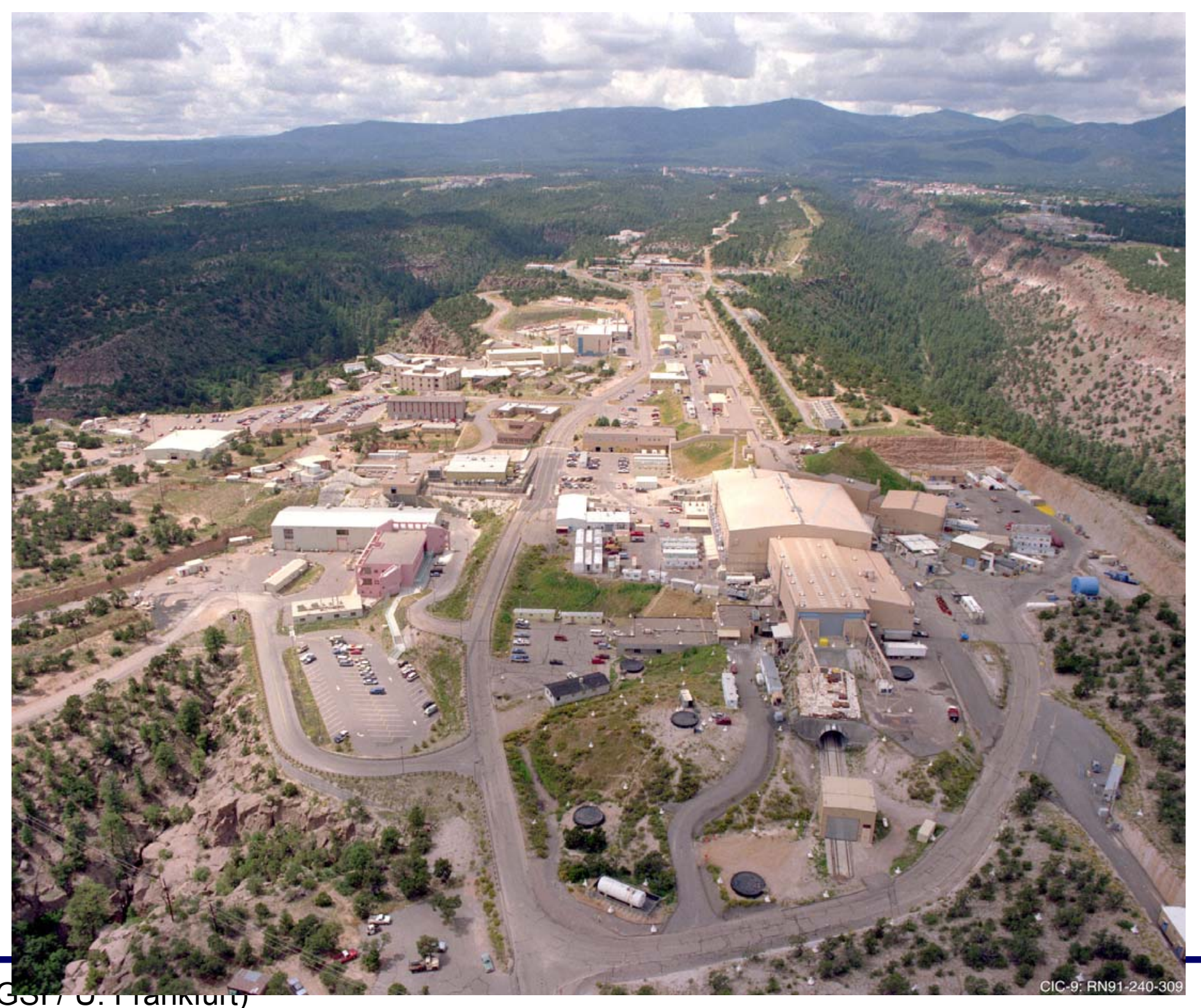

René Reifarth (Got, G. Traminti 


\section{Manuel Lujan Jr. Center}

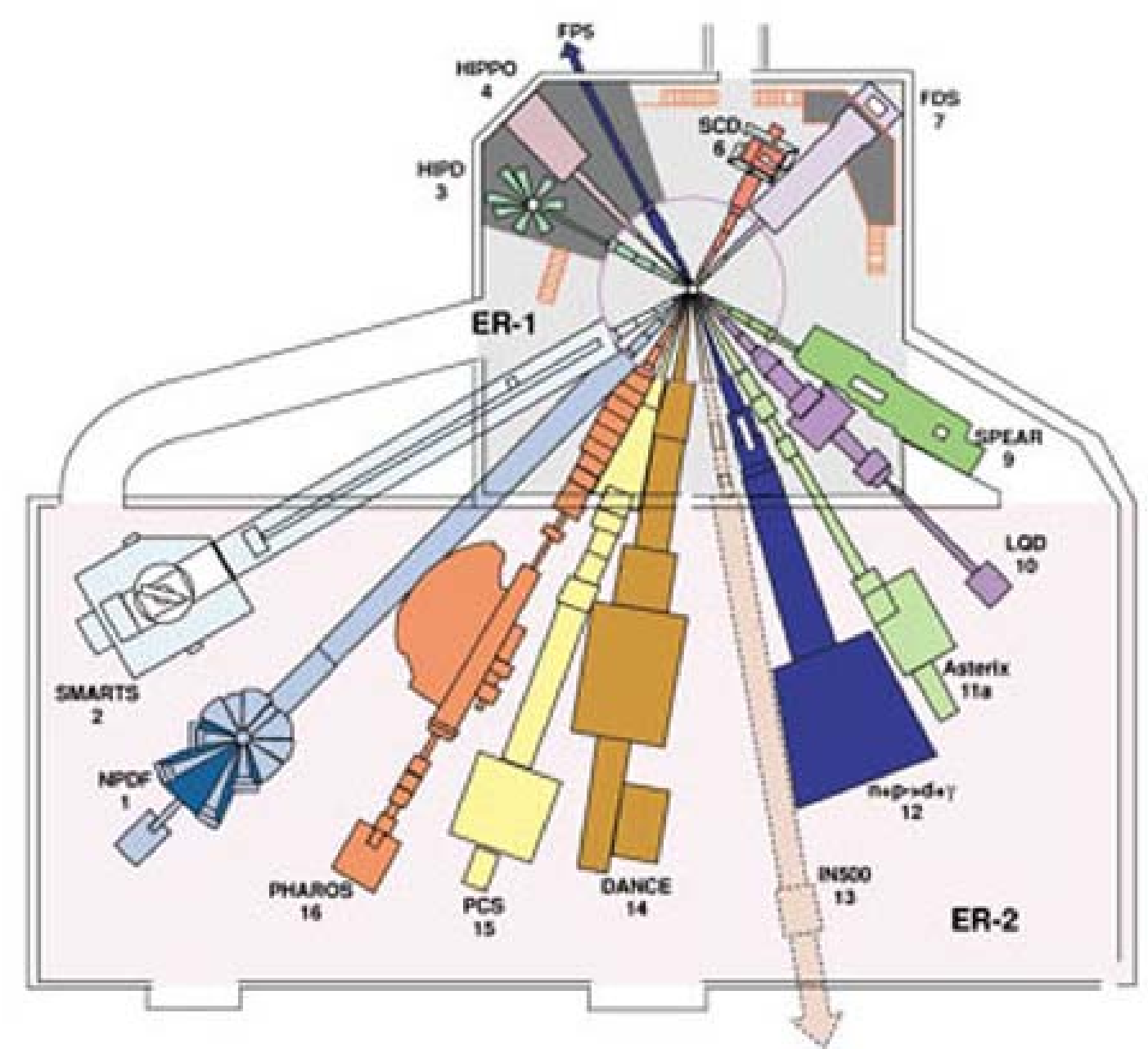

FP 14 views the second-tier coupled water moderator. 


\section{Neutron spectrum at spallation sources}

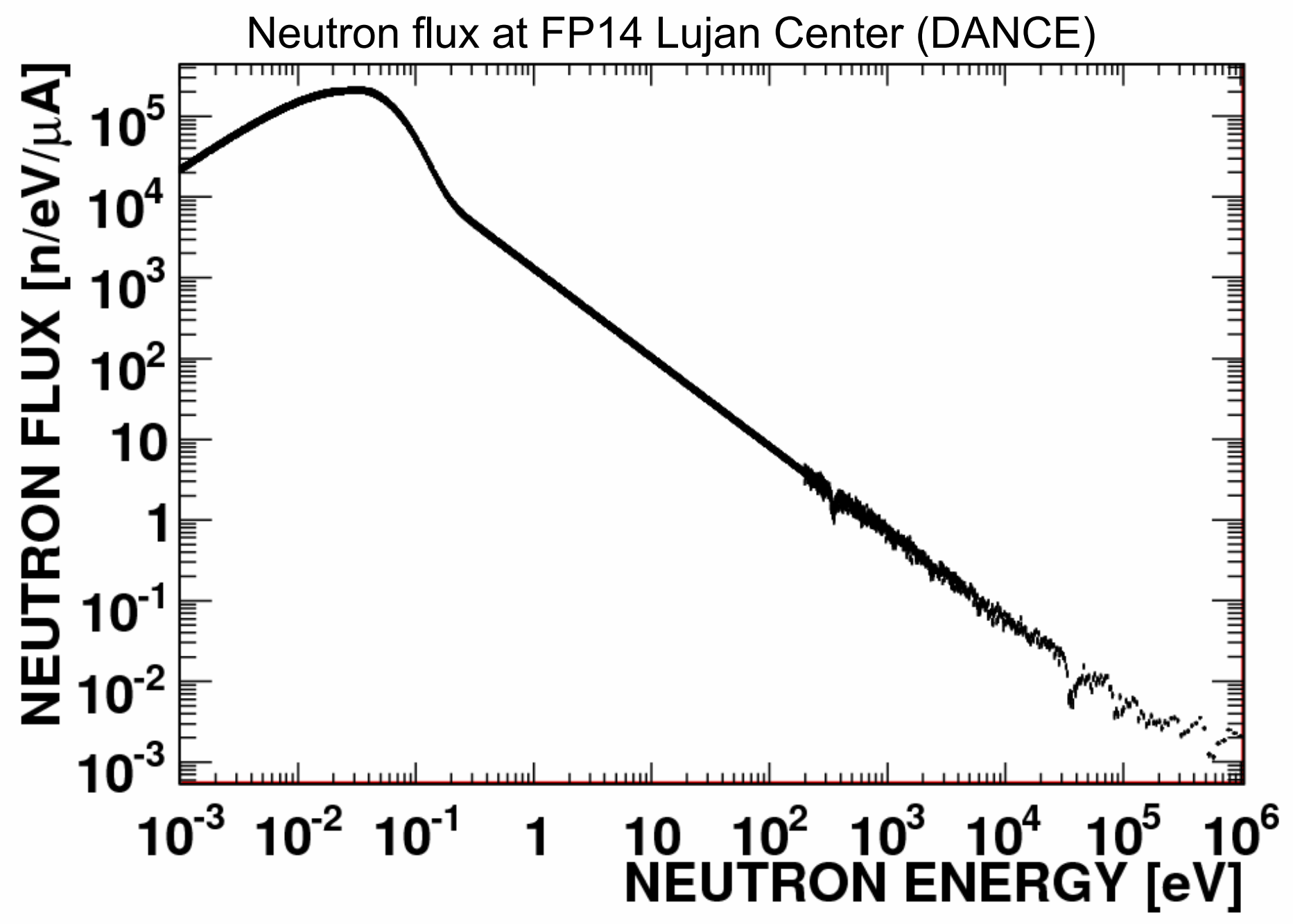

Conditions at LANSCE: $\Delta \mathrm{t}=0.1 \mu \mathrm{s}, \mathrm{E}_{\mathrm{p}}=800 \mathrm{MeV}, \mathrm{I}_{\mathrm{P}}=100 \mu \mathrm{A}(80 \mathrm{~kW})$ 


\section{The s-process around ${ }^{63} \mathrm{Ni}$}

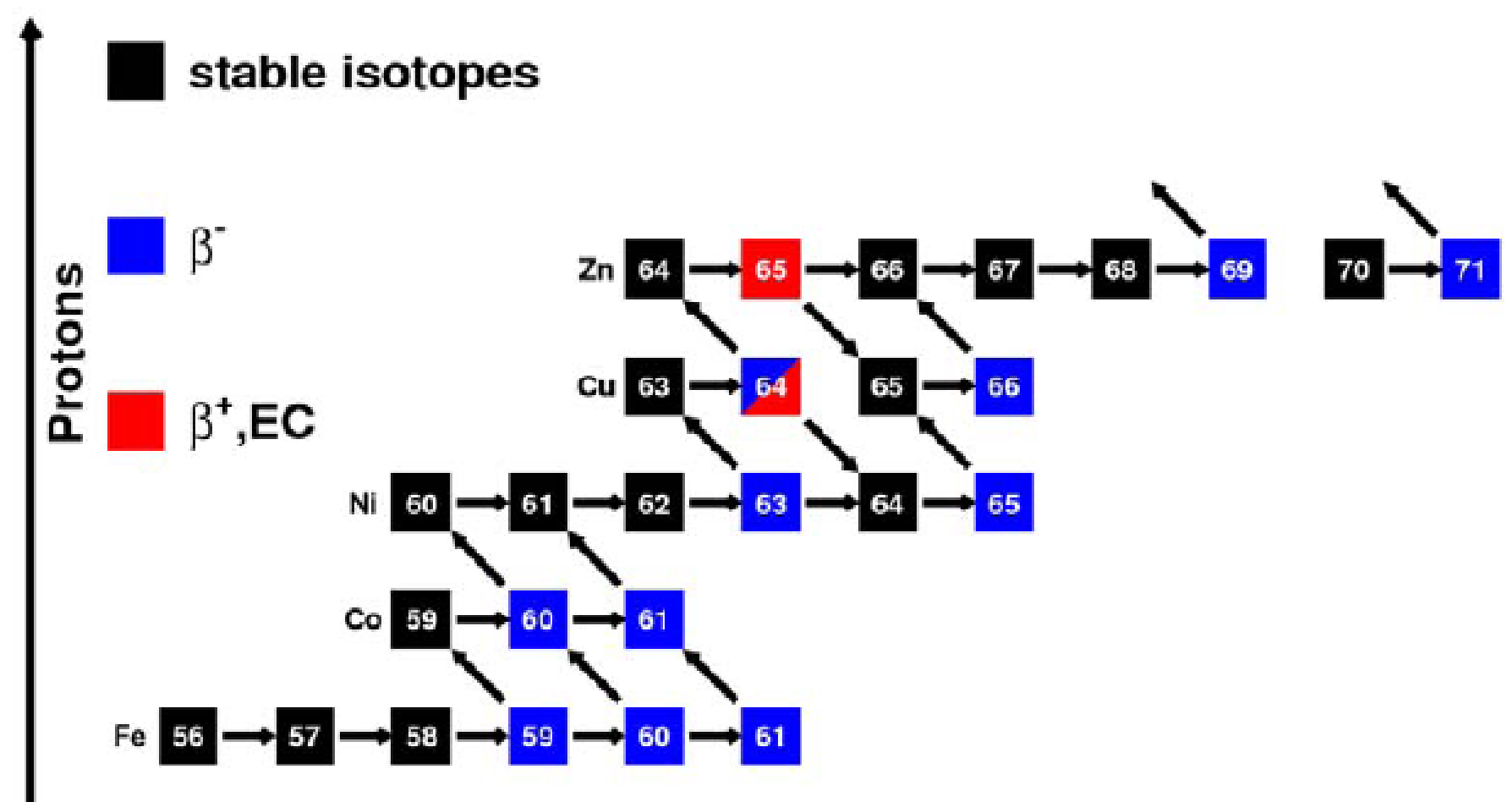

Neutrons

s-process nucleosynthesis in the region between iron and tin with the important branching at ${ }^{63} \mathrm{Ni}$ 


\section{Nuclear data needs for the weak s-process}
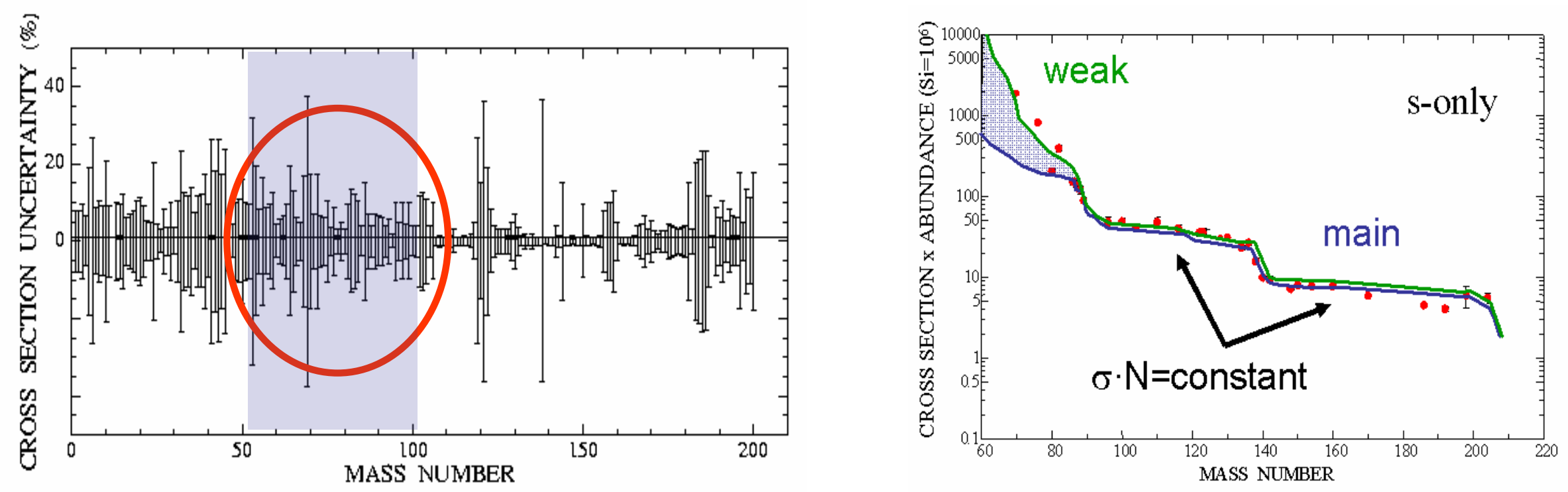

\section{Problems:}

- small cross sections

- resonance dominated

- contributions from direct capture

- propagation effects 


\section{Detector for Advanced}

\section{Neutron Capture Experiments}

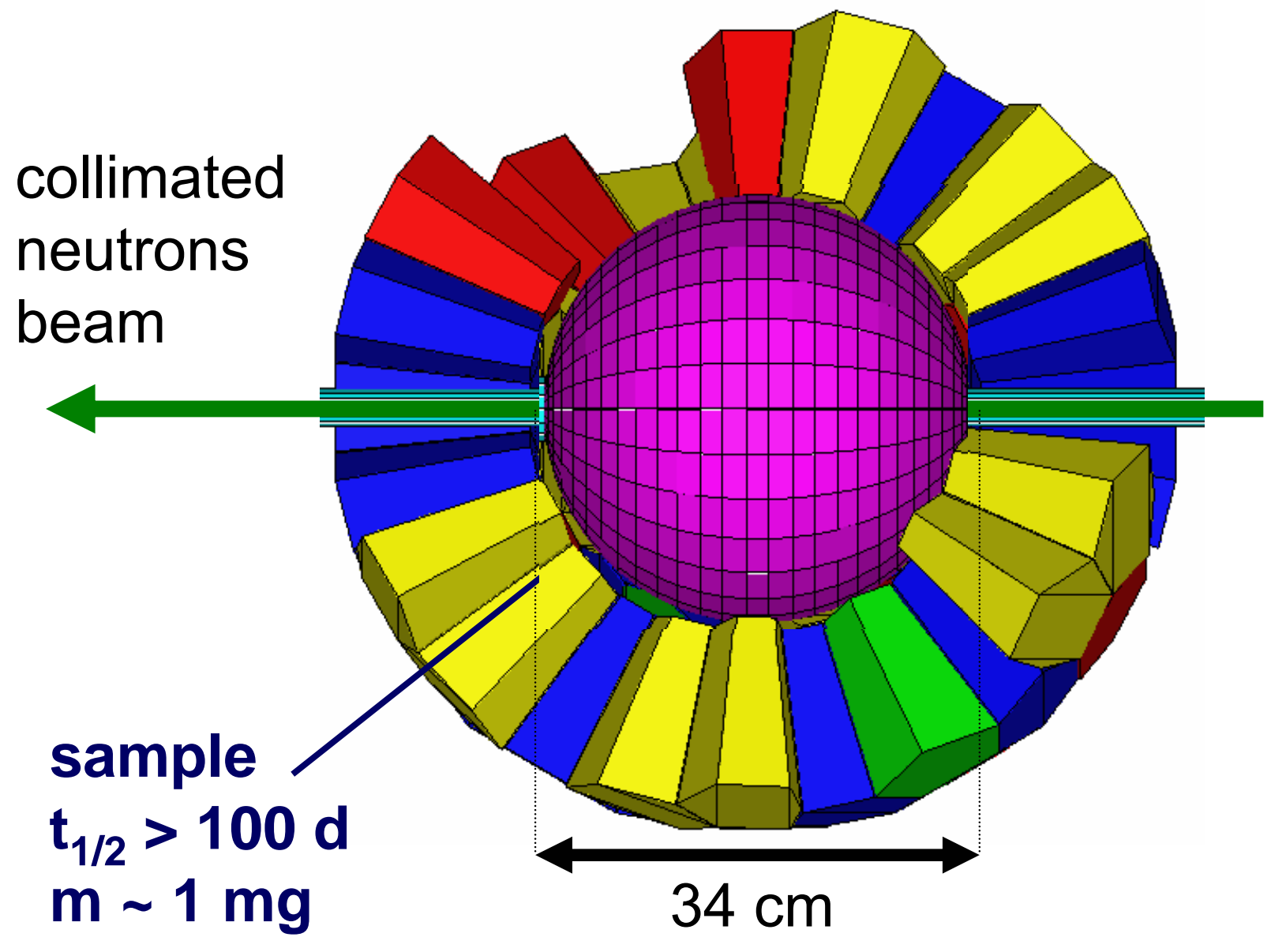

neutrons:

- spâllation source

- thermal .. $500 \mathrm{keV}$

- $20 \mathrm{~m}$ flight path

- $310^{5} \mathrm{n} / \mathrm{s} / \mathrm{cm}^{2} /$ decade

$\gamma$-Detector:

- $160 \mathrm{BaF}_{2}$ crystals

- 4 different shapes

- $R_{i}=17 \mathrm{~cm}, R_{a}=32 \mathrm{~cm}$

- $7 \mathrm{~cm}^{6} \mathrm{LiH}$ inside

- $\varepsilon_{\gamma} \approx 90 \%$

- $\varepsilon_{\text {casc }} \approx 98 \%$

René Reifarth (GSI / U. Frankfurt) 


\section{Background due to $(n, n)$}

\section{Reduction due to ${ }^{6} \mathrm{LiH}$ shell \\ $\left(\mathrm{R}_{\mathrm{i}}=10.5 \mathrm{~cm}, \mathrm{R}_{\mathrm{a}}=16.5 \mathrm{~cm}\right)$}
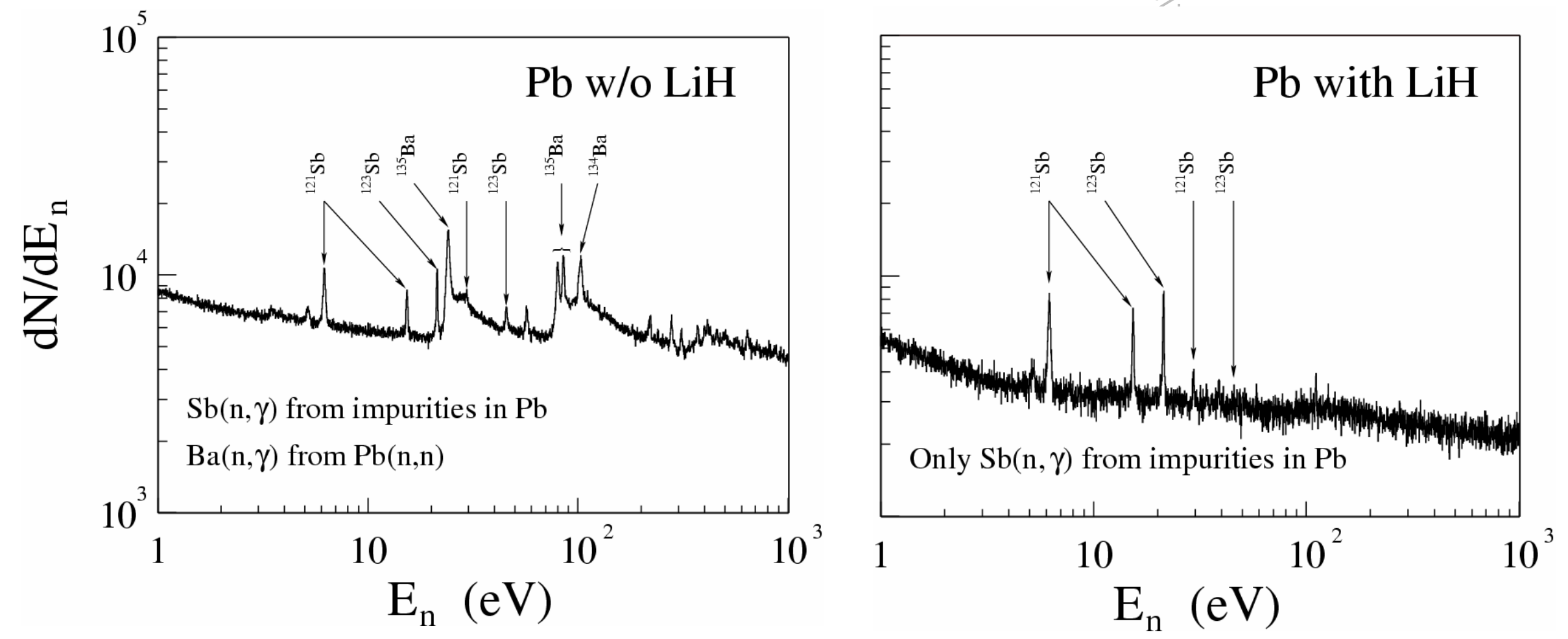

René Reifarth (GSI / U. Frankfurt) 


\section{Simulated effect of the ${ }^{6} \mathrm{LiH}$ absorber}

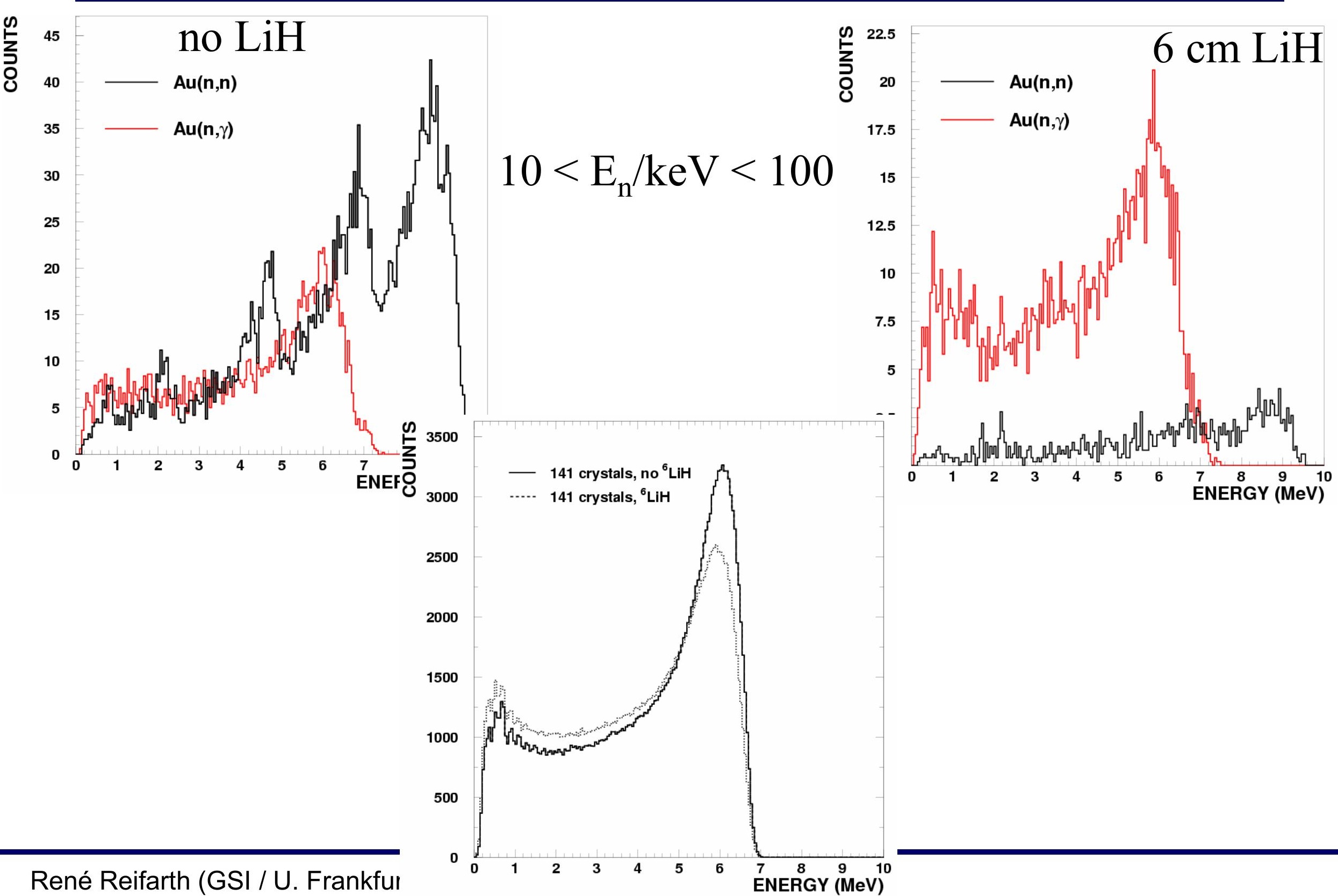




\section{Propagation effects in the weak s-process}

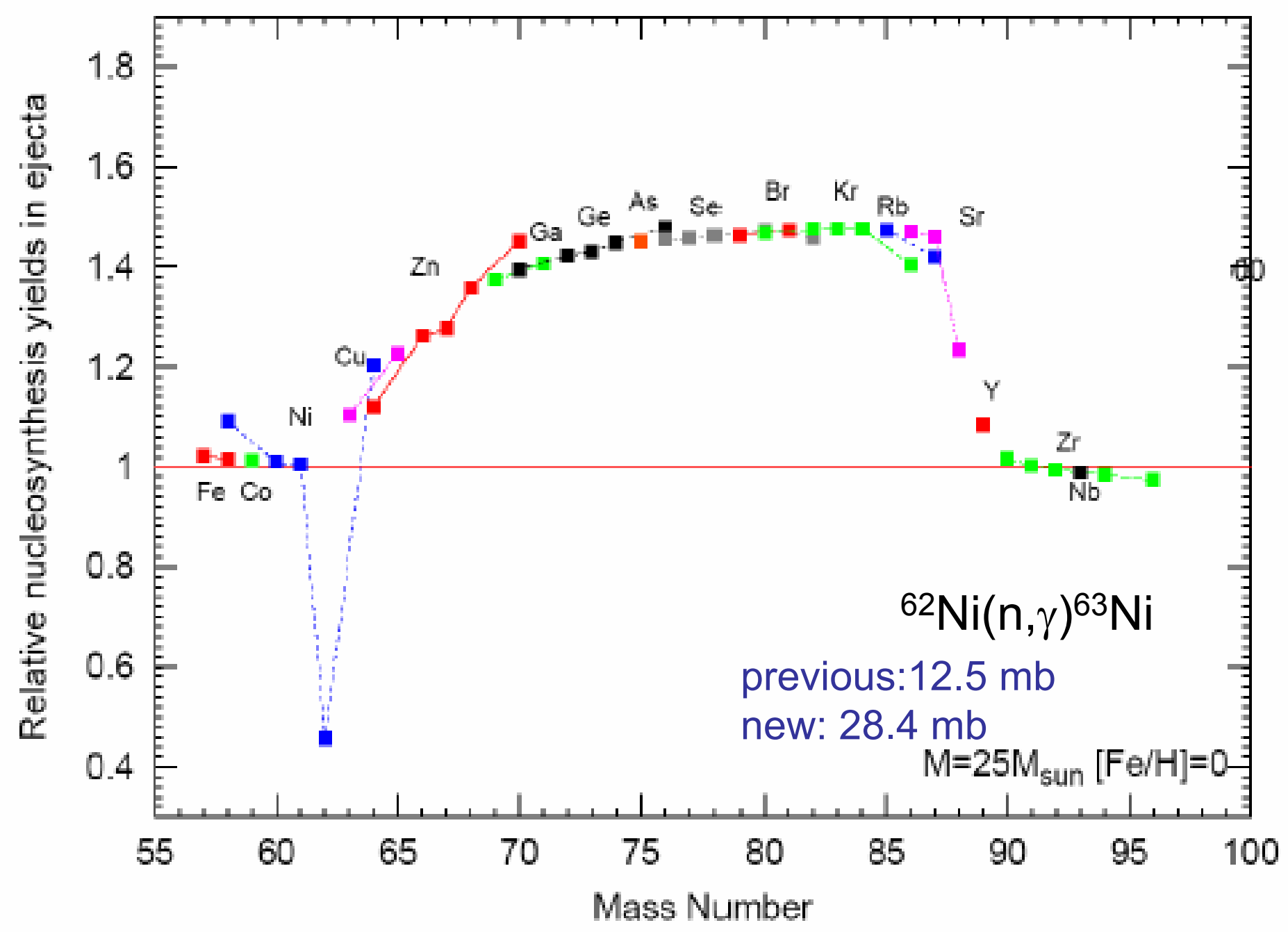

Nassar et al., Phys. Rev. Lett. 94, 092504 (2005)

René Reifarth (GSI / U. Frankfurt) 


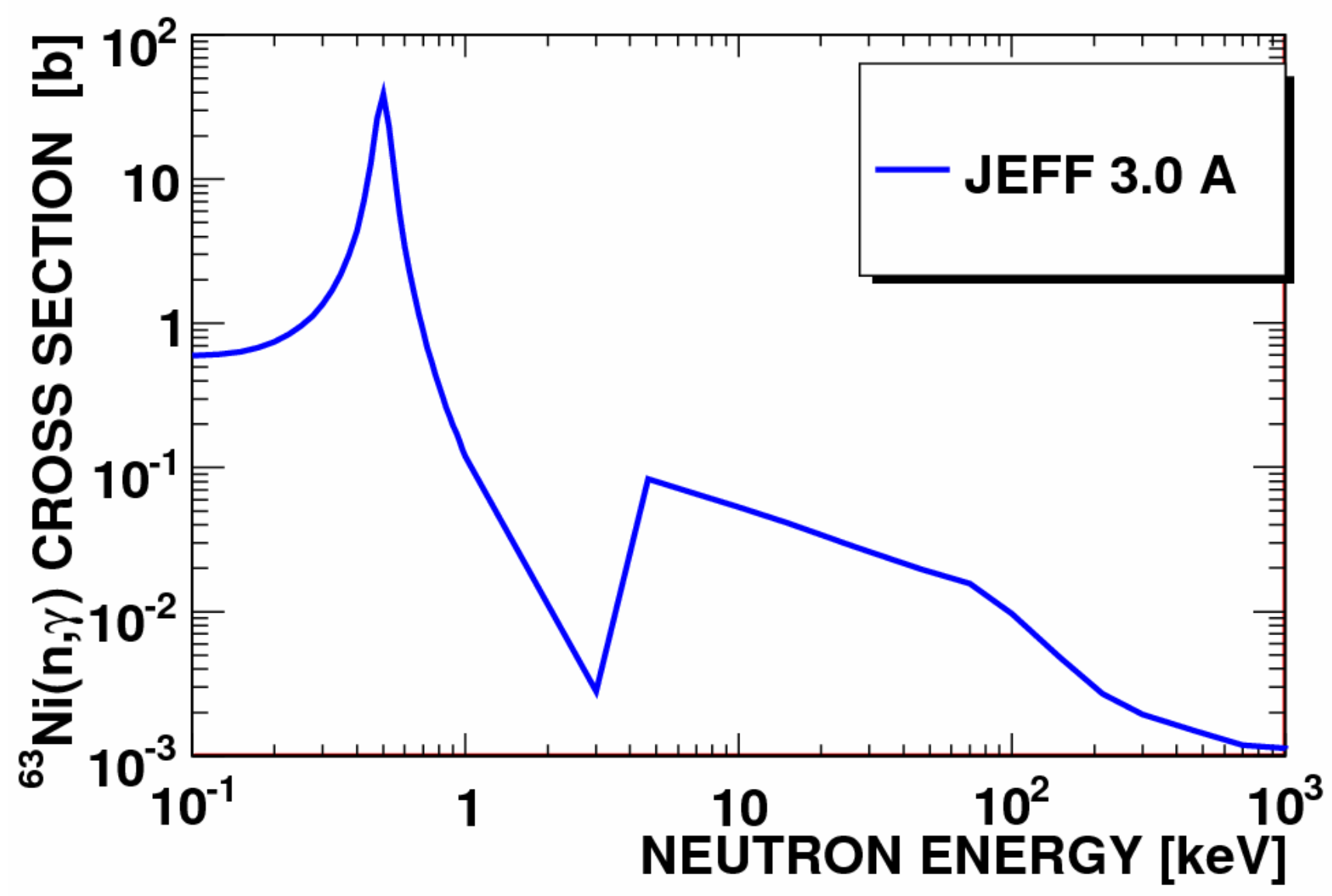

No experimental data exist so far (only transmission measurements) 


\section{Evidence for neutron capture: INDIRECT}

$$
\begin{aligned}
& \mathrm{A} X+n \Rightarrow \mathrm{A}^{+1} \mathrm{X} \\
& { }^{\mathrm{A}+1} \mathrm{X} \Rightarrow \mathrm{A}^{+1} \mathrm{Y}+\gamma+\ldots
\end{aligned}
$$

Produced Activity:

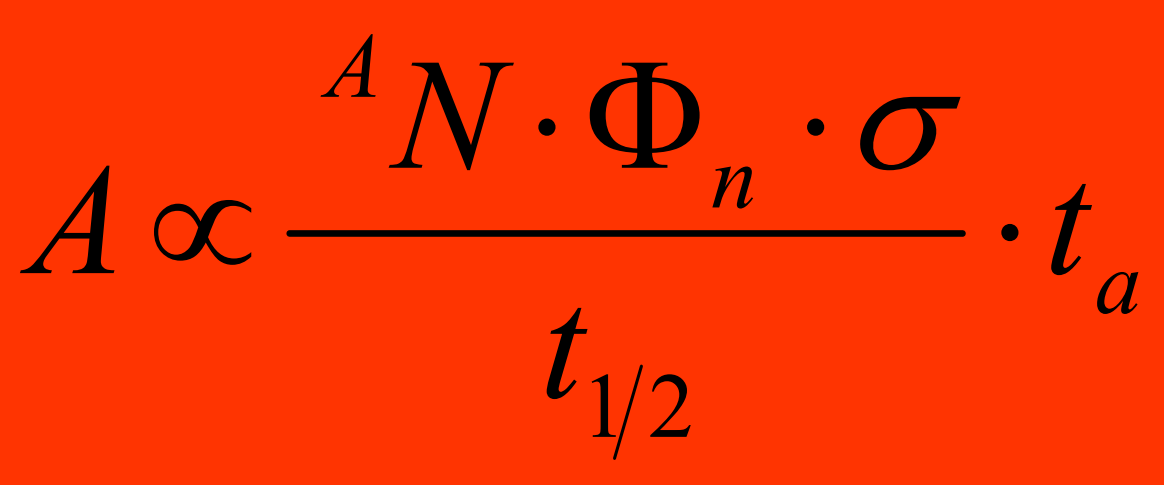

René Reifarth (GSI / U. Frankfurt) 


\section{Neutron Capture on ${ }^{14} \mathrm{C}$}

- Verification of Coulomb Dissociation (CD) as an indirect method for determining $(n, \gamma)$ rates

- Big Bang Nucleosynthesis

- Neutron-induced CNO cycles - s-process

- Neutrino-driven winds - r-process

$\mathbf{O}$

$\mathbf{N}$

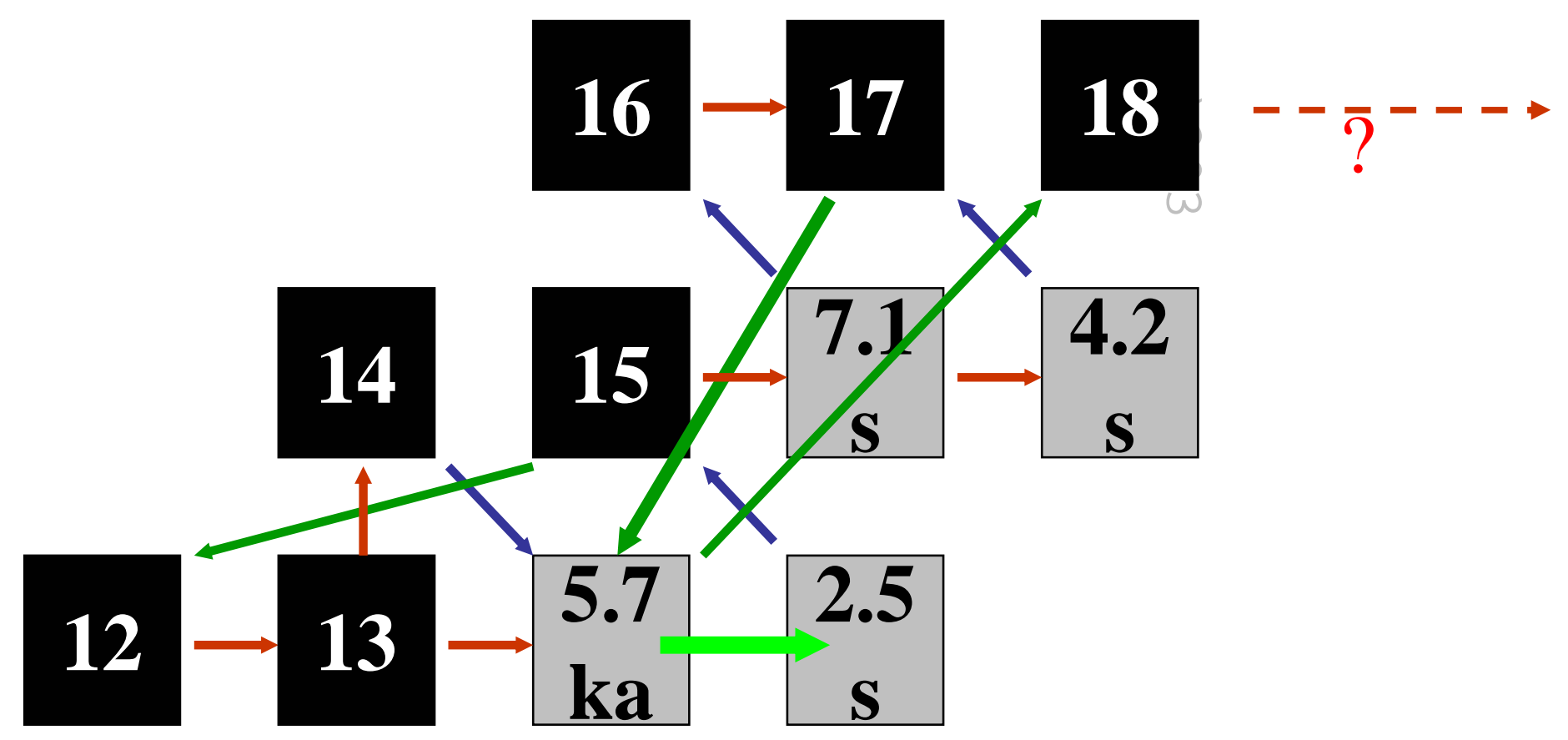




\section{${ }^{14} \mathrm{C}$ - sample}

- $283 \mathrm{mg}{ }^{14} \mathrm{C}\left(\mathrm{t}_{1 / 2}=5.7 \mathrm{ka}\right)$, determined from decay heat

- carrier: ${ }^{\text {nat }} \mathrm{C}$, activated $\mathrm{Ni}$-container

- active impurities:

$$
-{ }^{44} \mathrm{Ti}\left(\mathrm{t}_{1 / 2}=44 \mathrm{y}\right)
$$

- $21 \times 12 \mathrm{~mm}^{2}$ diameter

\section{activation only (presently) feasible method}




\section{Activation Method}

${ }^{14} \mathrm{C}(\mathrm{n}, \gamma){ }^{15} \mathrm{C}$ reaction detected via ${ }^{15} \mathrm{C}\left(\beta^{-}\right){ }^{15} \mathrm{~N}$ decay

$$
\left(\mathrm{t}_{1 / 2}=2.5 \mathrm{~s}\right)
$$

Determination of neutron flux via ${ }^{197} \mathrm{Au}(\mathrm{n}, \gamma){ }^{198} \mathrm{Au}$

Neutron source:

$$
{ }^{7} \mathrm{Li}(\mathrm{p}, \mathrm{n}){ }^{7} \mathrm{Be}
$$

${ }^{14} \mathrm{C}$ sample irradiated for $10 \mathrm{~s}$, then activity counted for $10 \mathrm{~s}$ („cyclic activation“)

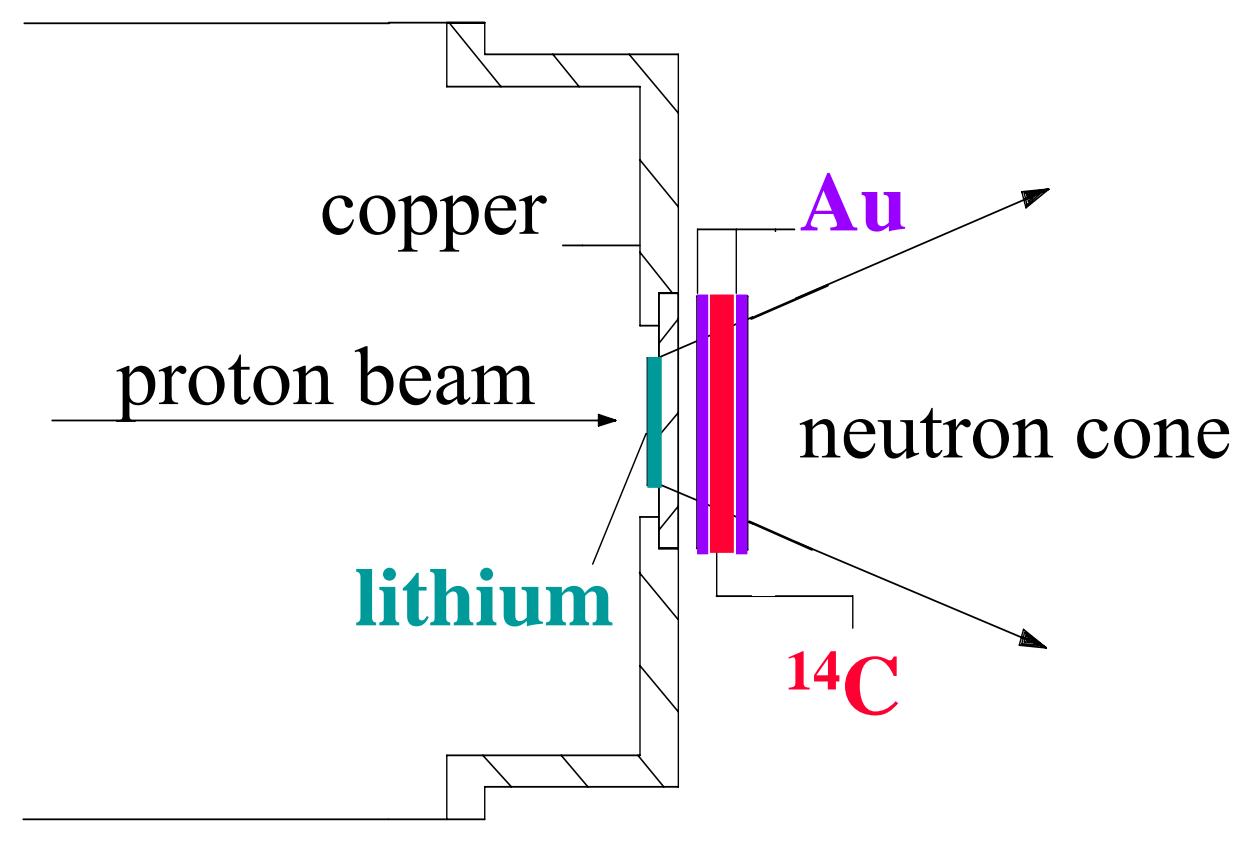

R. Reifarth et. al, PRC C 77, 015804 (2008) 


\section{A standard neutron spectrum - working horse!}

$E_{p}=1912 \mathrm{keV}$, neutron cone fully covered

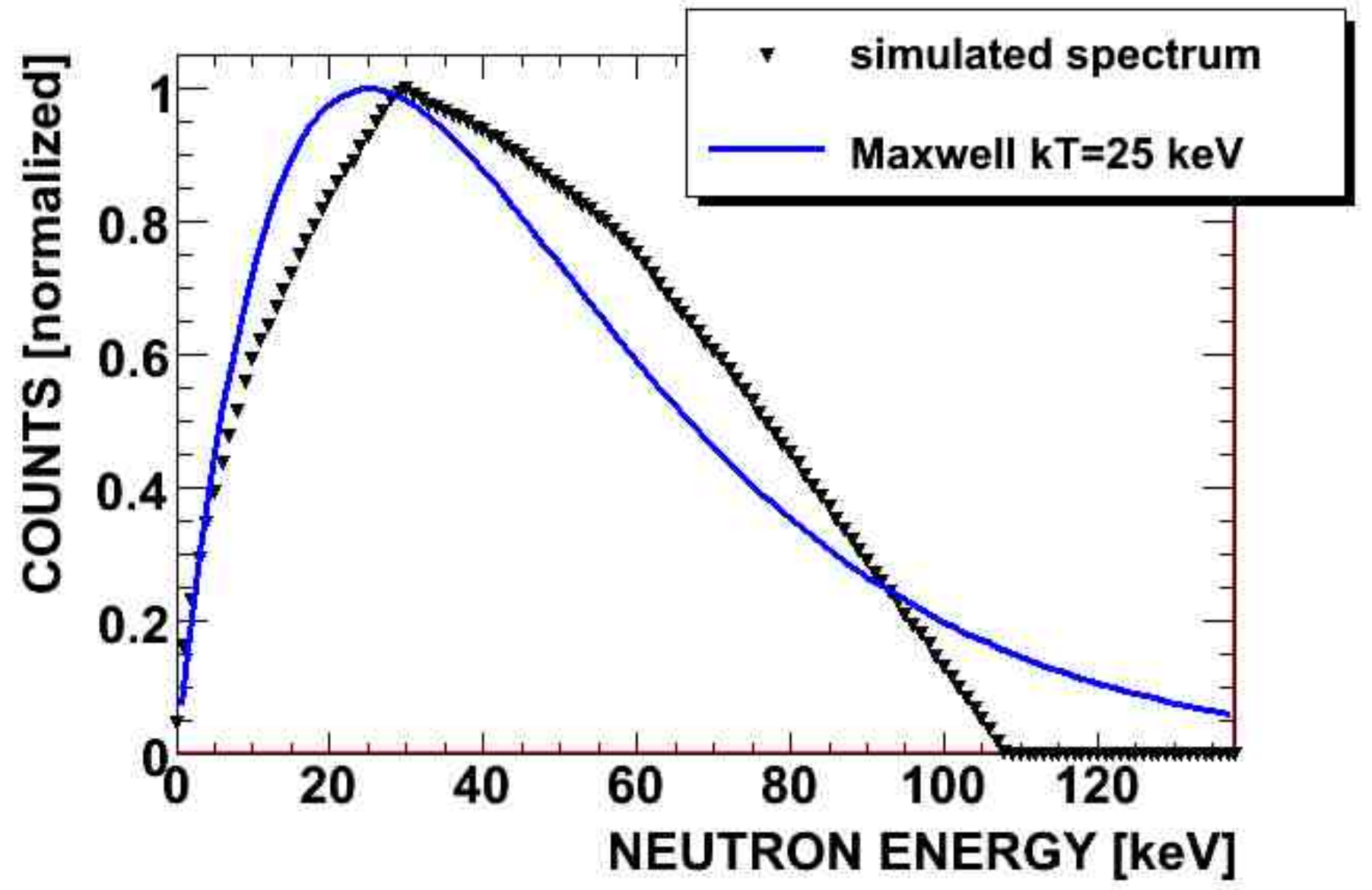
Quasi-Maxwellian
$k T=25 \mathrm{keV}$
averaged distribution:
$E_{\text {max }}=110 \mathrm{keV}$ 


\section{Other neutron spectra}
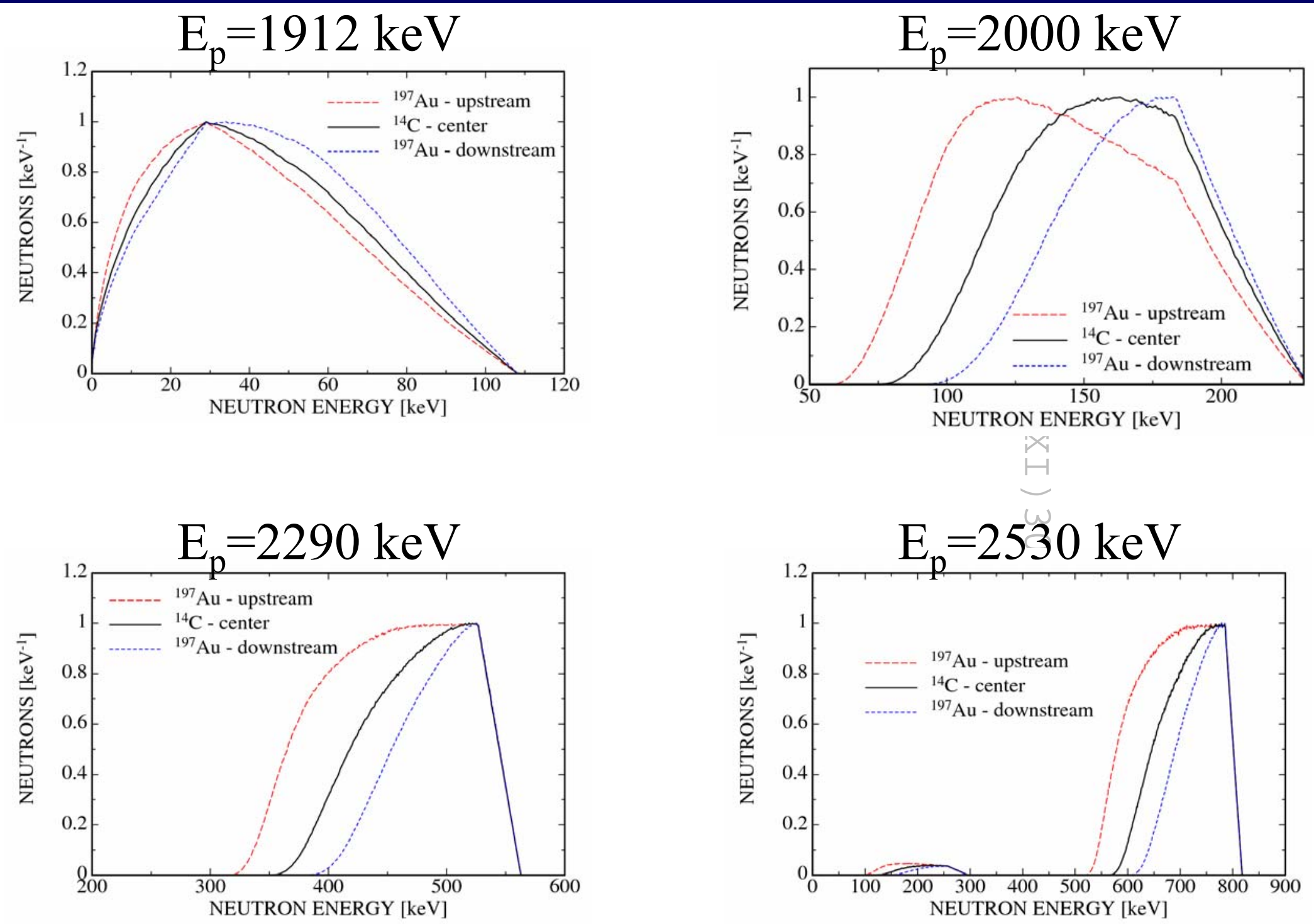

René Reifarth (GSI / U. Frankfurt) 


\section{${ }^{15} \mathrm{C}-\gamma$-spectra}
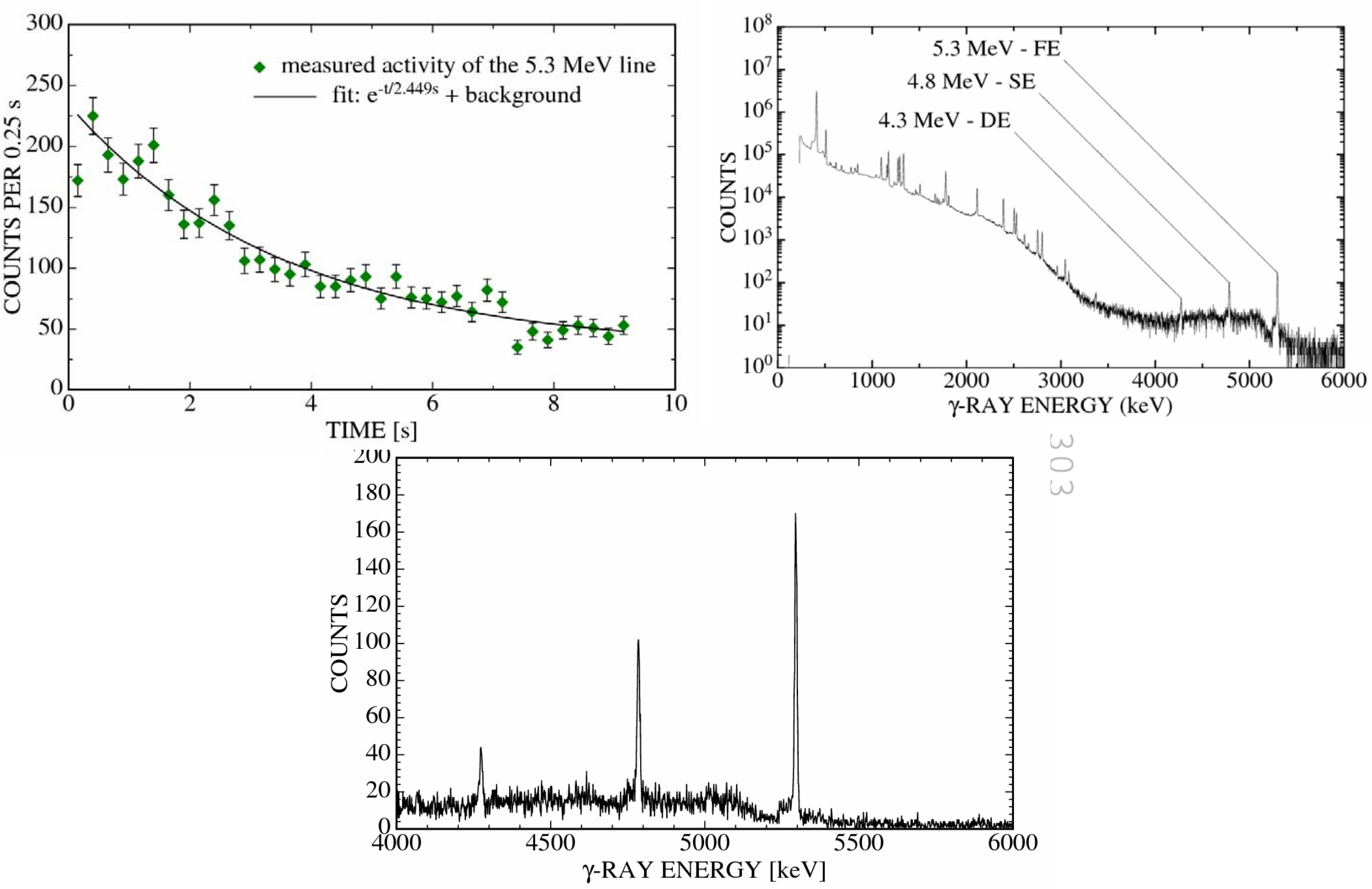

René Reifarth (GSI / U. Frankfurt) 


\section{Description and Deconvolution}

\begin{tabular}{|c|c|c|c|}
\hline 15 & 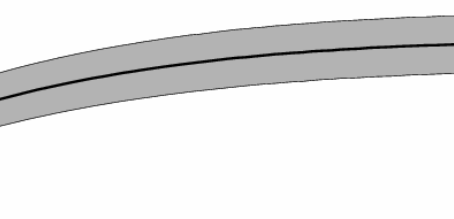 & \multicolumn{2}{|c|}{$\begin{array}{l}\text { - p-wave capture } \\
\text { - good agreement with exp. } d\end{array}$} \\
\hline${ }_{100} \quad 200300$ & 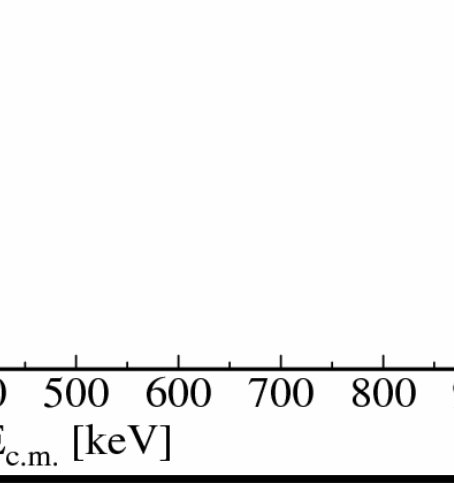 & & 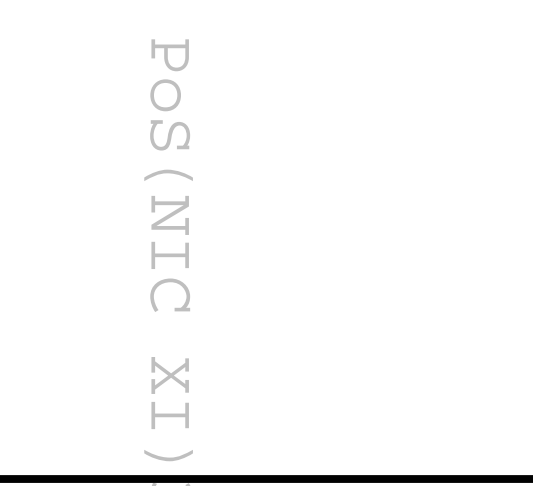 \\
\hline keV & Exp. $[\mu b]$ & Theo. [ub] & Theo/Exp \\
\hline 23 & $7.1 \pm 5$ & $6.5 \pm 0.4$ & $0.92 \pm 0.08$ \\
\hline 150 & $10.7 \pm 1.2$ & $11.7 \pm 0.6$ & $1.09 \pm 0.12$ \\
\hline 500 & $17.0 \pm 1.5$ & $16.5 \pm 0.8$ & $0.97 \pm 0.10$ \\
\hline 800 & $15.8 \pm 1.6$ & $17.5 \pm 0.9$ & $1.11 \pm 0.11$ \\
\hline
\end{tabular}




\section{Comparison with other rate estimates}

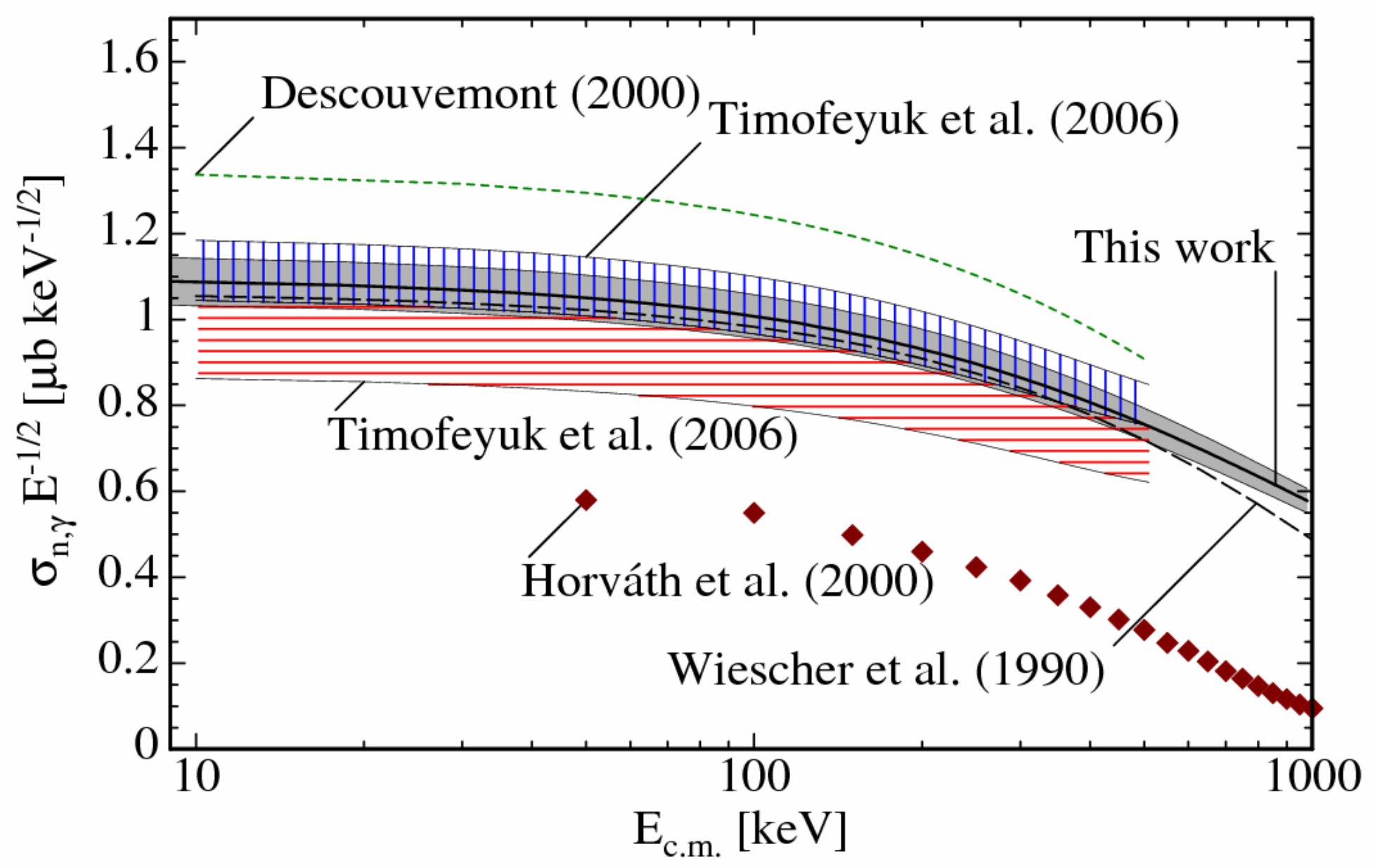

René Reifarth (GSI / U. Frankfurt) 


\section{Comparison with CD}

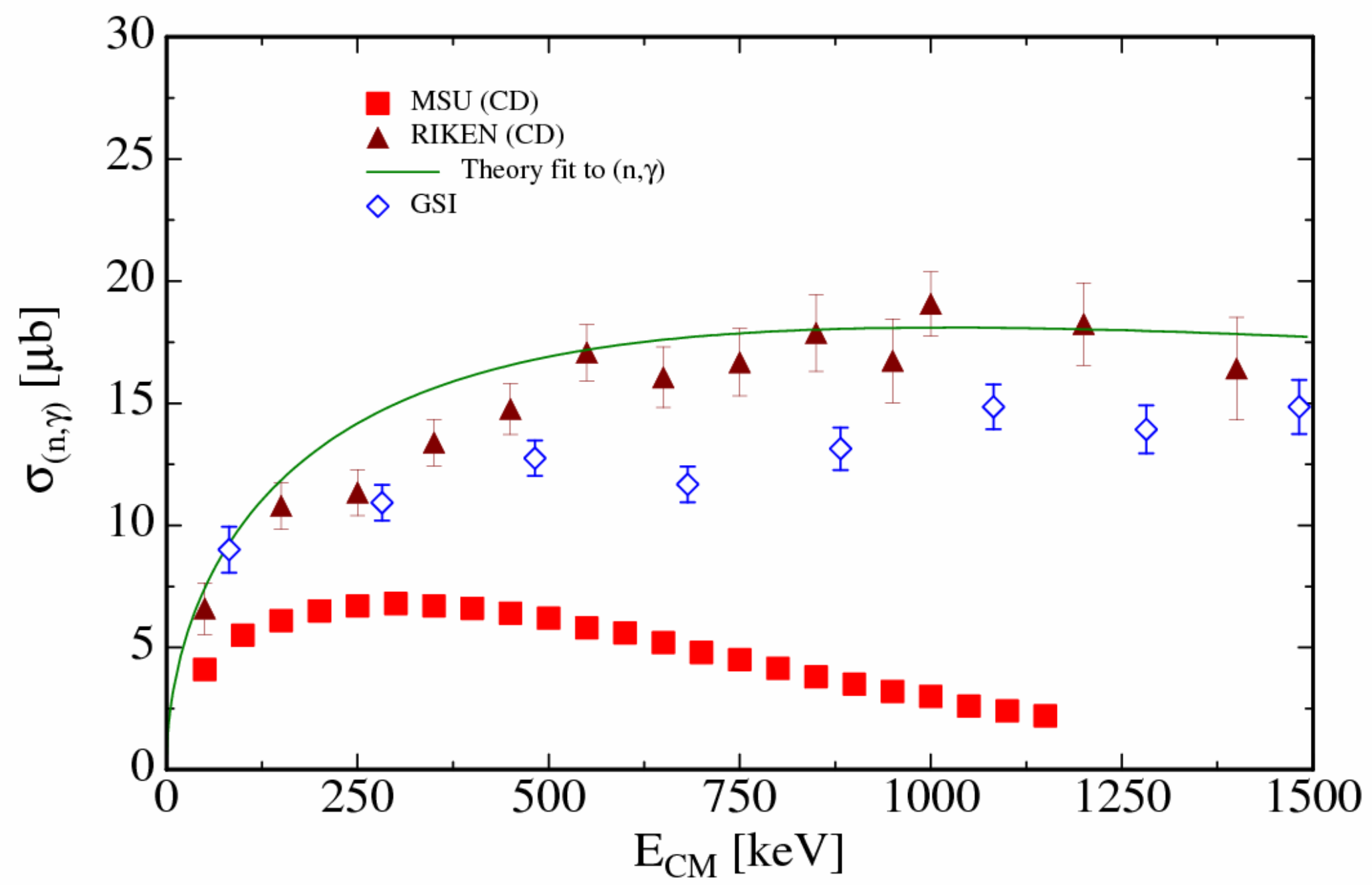

René Reifarth (GSI / U. Frankfurt) 


\section{Future developments}

- Ever more neutrons

- Indirect methods 


\section{The Frankfurt neutron source at the Stern-Gerlach-Zentrum (FRANZ)}

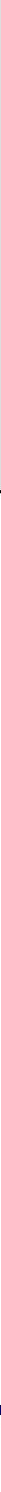




\section{Possible experimental program at FRANZ and ESS}

The Frankfurt neutron source will provide the highest neutron flux for a nuclear astrophysics program in relevant keV region $(1-500 \mathrm{keV})$ worldwide.

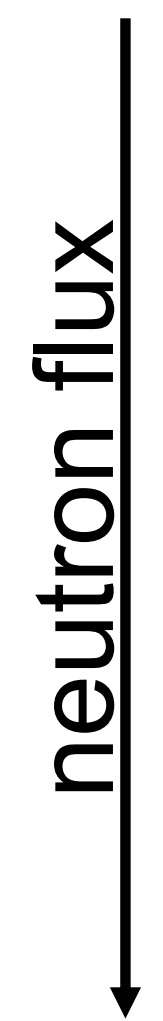

\section{Neutron capture measurements of small cross sections:}

- Big Bang nucleosynthesis: ${ }^{1} \mathrm{H}(\mathrm{n}, \gamma)$

- Neutron poisons for the s-process: ${ }^{12} \mathrm{C}(\mathrm{n}, \gamma),{ }^{16} \mathrm{O}(\mathrm{n}, \gamma),{ }^{22} \mathrm{Ne}(\mathrm{n}, \gamma)$.

- ToF measurements of medium mass nuclei for the weak s-process.

\section{Neutron capture measurements with small sample masses:}

- Radio-isotopes for $\gamma$-ray astronomy ${ }^{59} \mathrm{Fe}(\mathrm{n}, \gamma)$ and ${ }^{60} \mathrm{Fe}(\mathrm{n}, \gamma)$

- Branch point nuclei, e.g. ${ }^{85} \mathrm{Kr}(\mathrm{n}, \gamma),{ }^{95} \mathrm{Zr}(\mathrm{n}, \gamma),{ }^{147} \mathrm{Pm}(\mathrm{n}, \gamma)$,

$$
{ }^{154} \operatorname{Eu}(\mathrm{n}, \gamma),{ }^{155} \mathrm{Eu}(\mathrm{n}, \gamma),{ }^{153} \mathrm{Gd}(\mathrm{n}, \gamma),{ }^{185} \mathrm{~W}(\mathrm{n}, \gamma)
$$




\section{Setup with very short flight path}
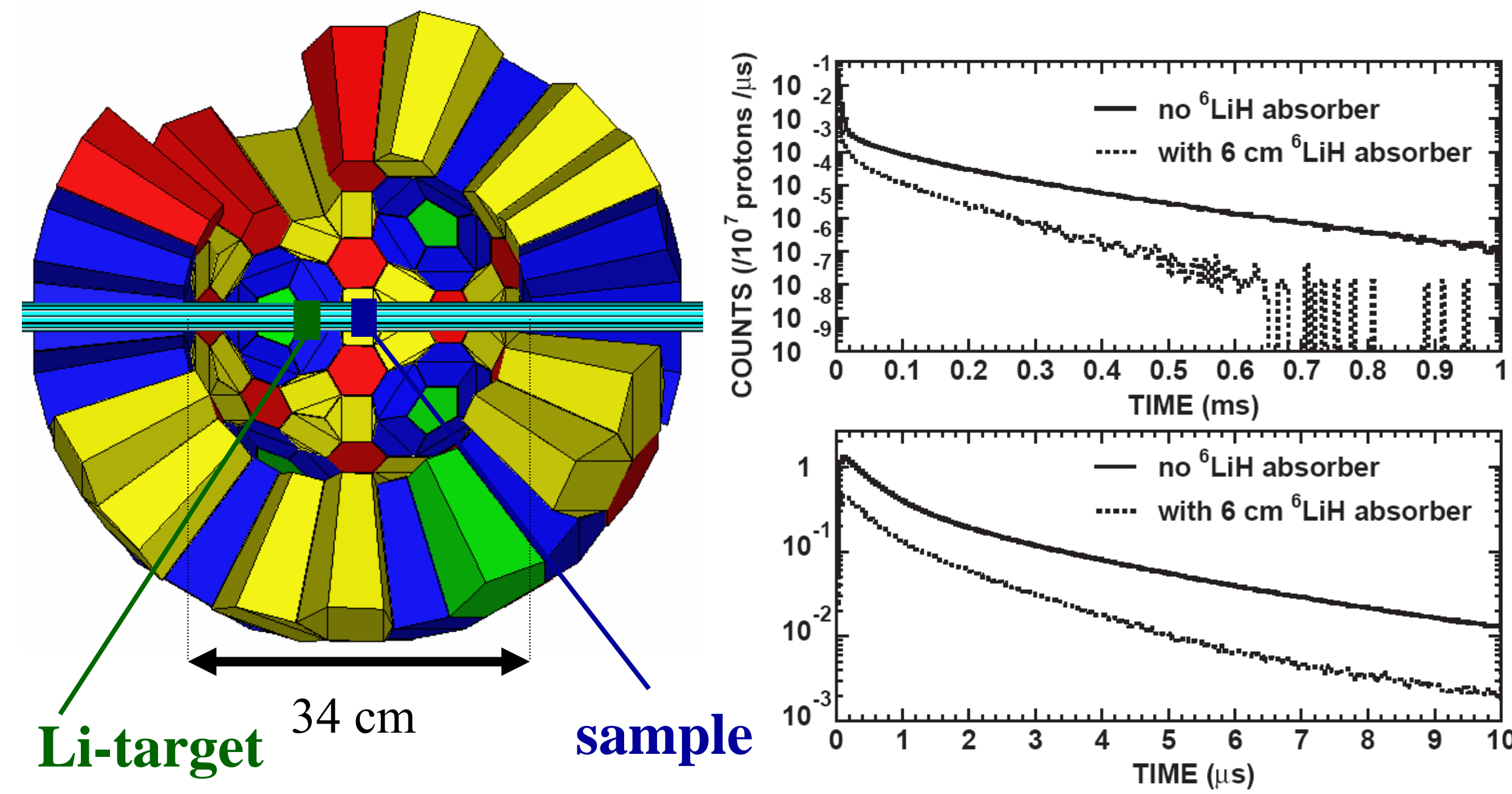

Challenge: Neutrons bouncing around in the detector

René Reifarth (GSI / U. Frankfurt) 


\section{TOF spectrum-very short flight path}

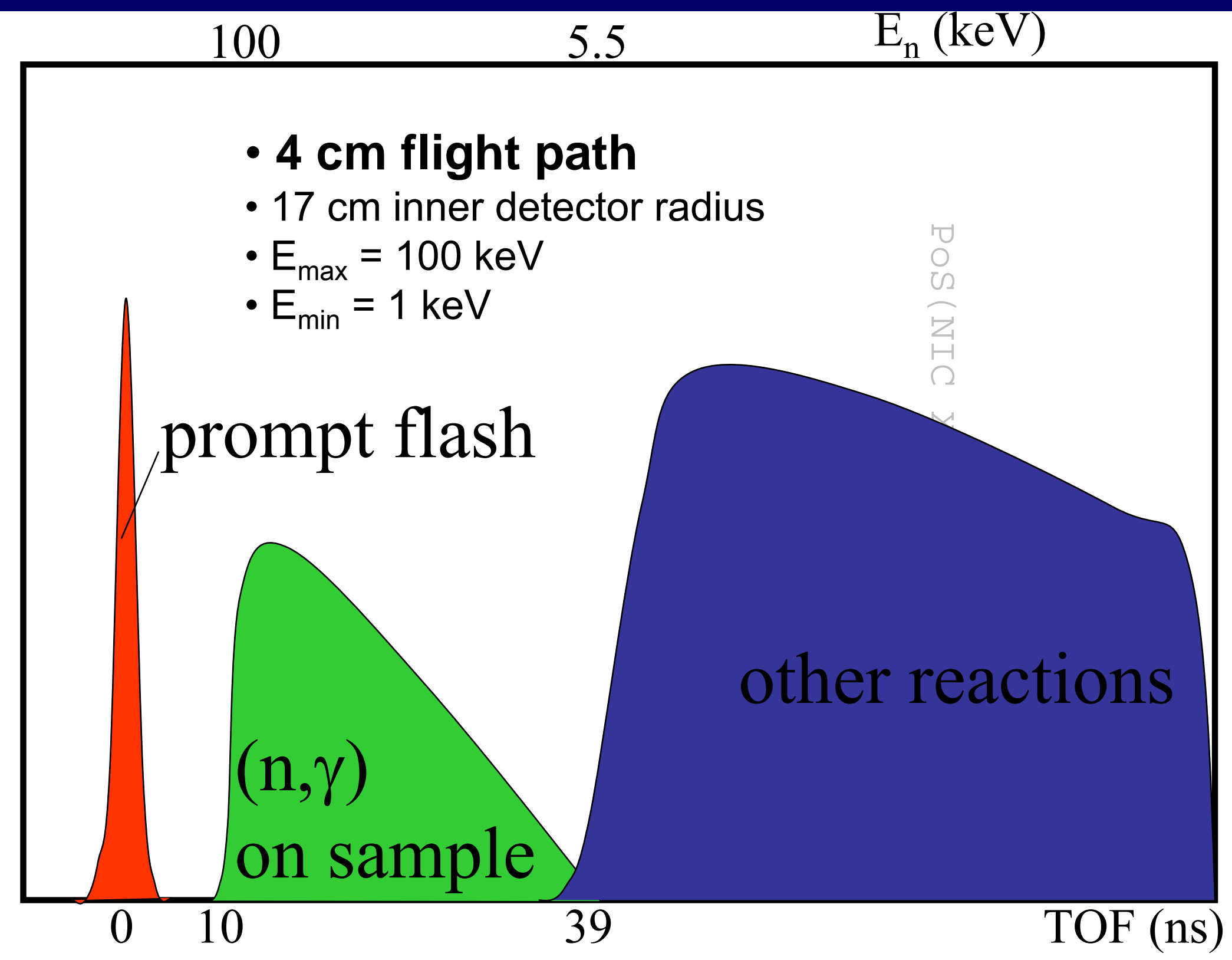

René Reifarth (GSI / U. Frankfurt) 


\section{Motivation $-{ }^{60} \mathrm{Fe}$ in the universe}

Detection of $\gamma$-ray lines from interstellar ${ }^{60} \mathrm{Fe}$ with SPI (INTEGRAL)

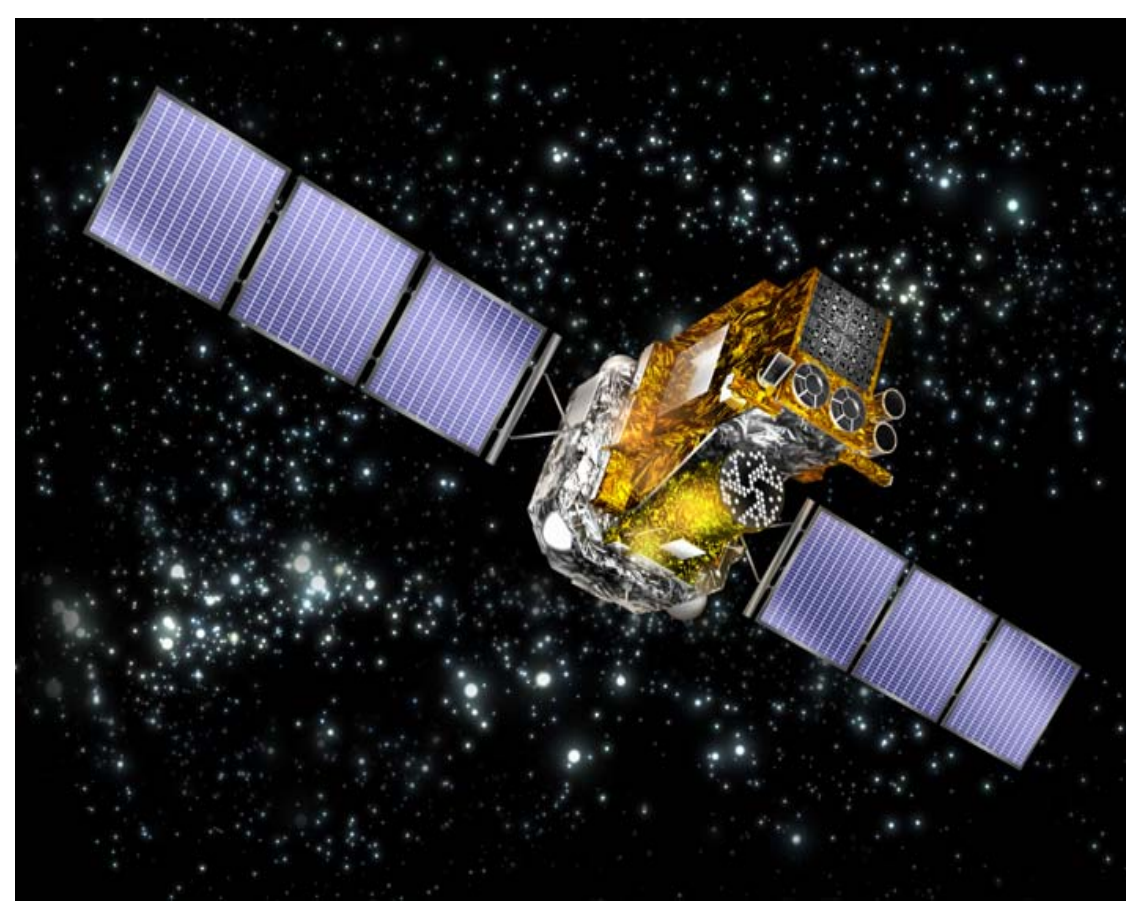

$$
\mathrm{E}_{\gamma}=1173 \text { and } 1333 \mathrm{keV}
$$

ongoing production in massive stars and

distribution by subsequent supernovae

tests stellar model and SN rate

Harris et al, A\&A 433 (2005) L49 
- can be found in deep sea manganese crusts

- Gives hints about a nearby supernova

- $2.8 \mathrm{Ma}$ ago
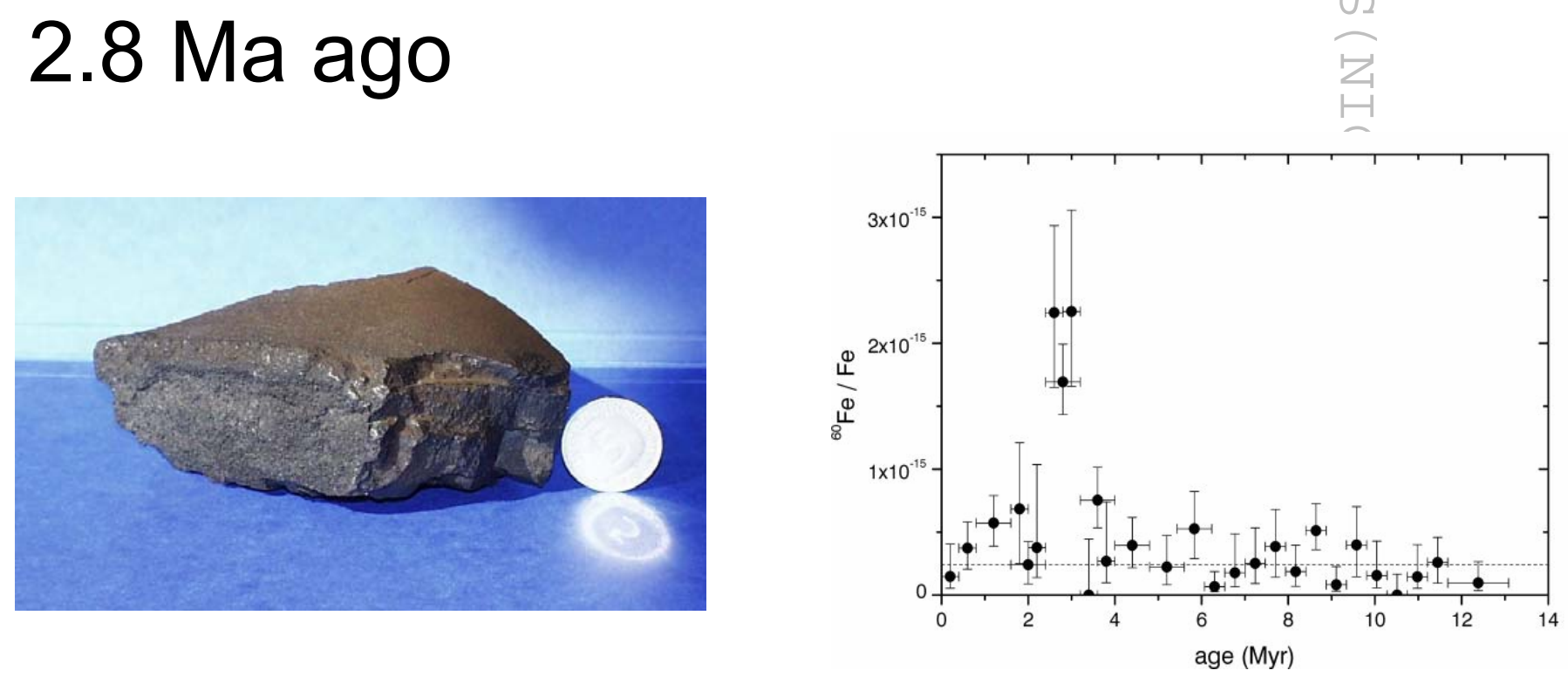

Knie et al, PRL 93 (2004) 171103 


\section{${ }^{60} \mathrm{Fe}$ in stars}

- Weak s-process component

- During C-shell burning in massive stars

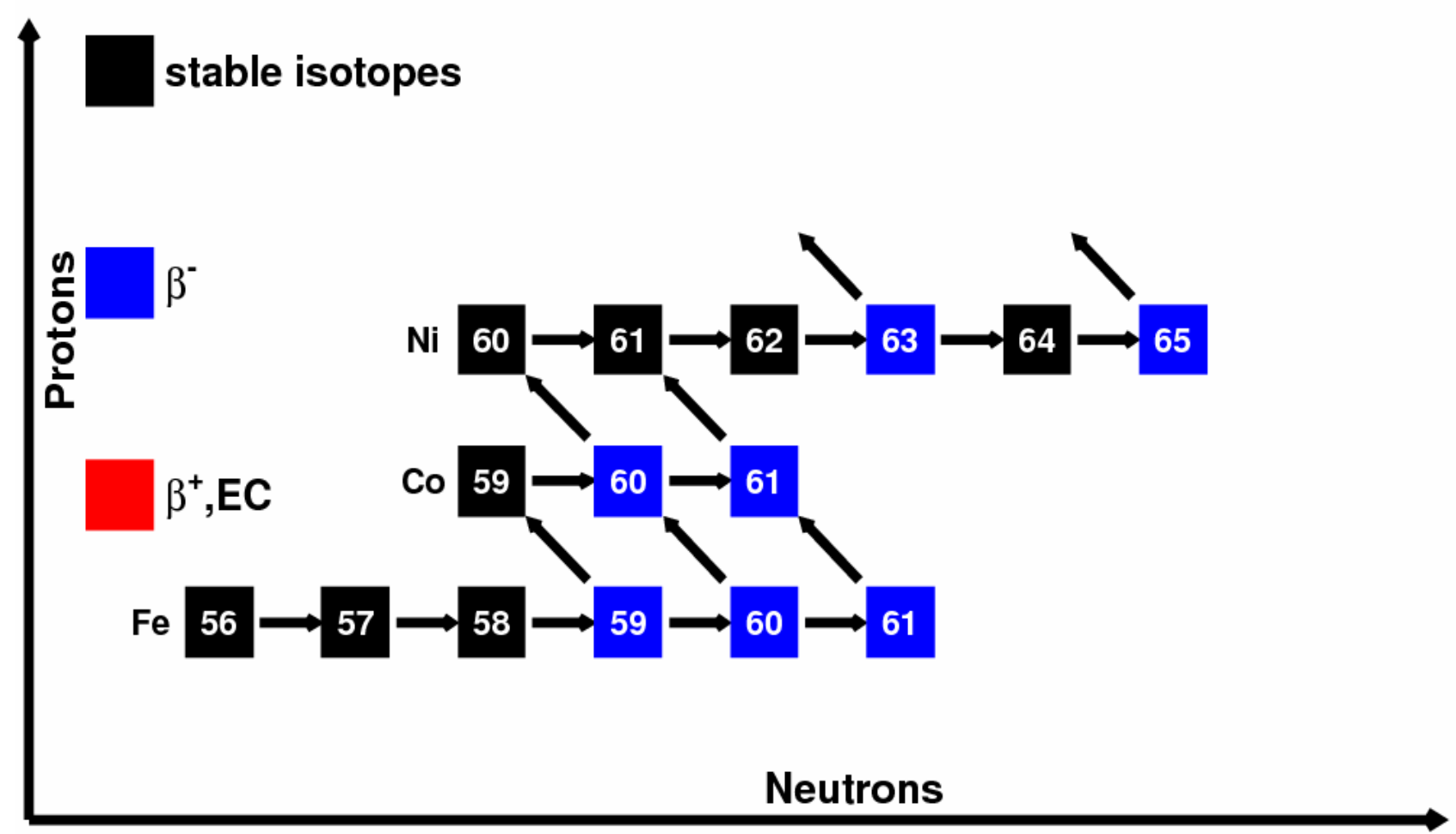




\section{Production and Destruction of ${ }^{60} \mathrm{Fe}$}

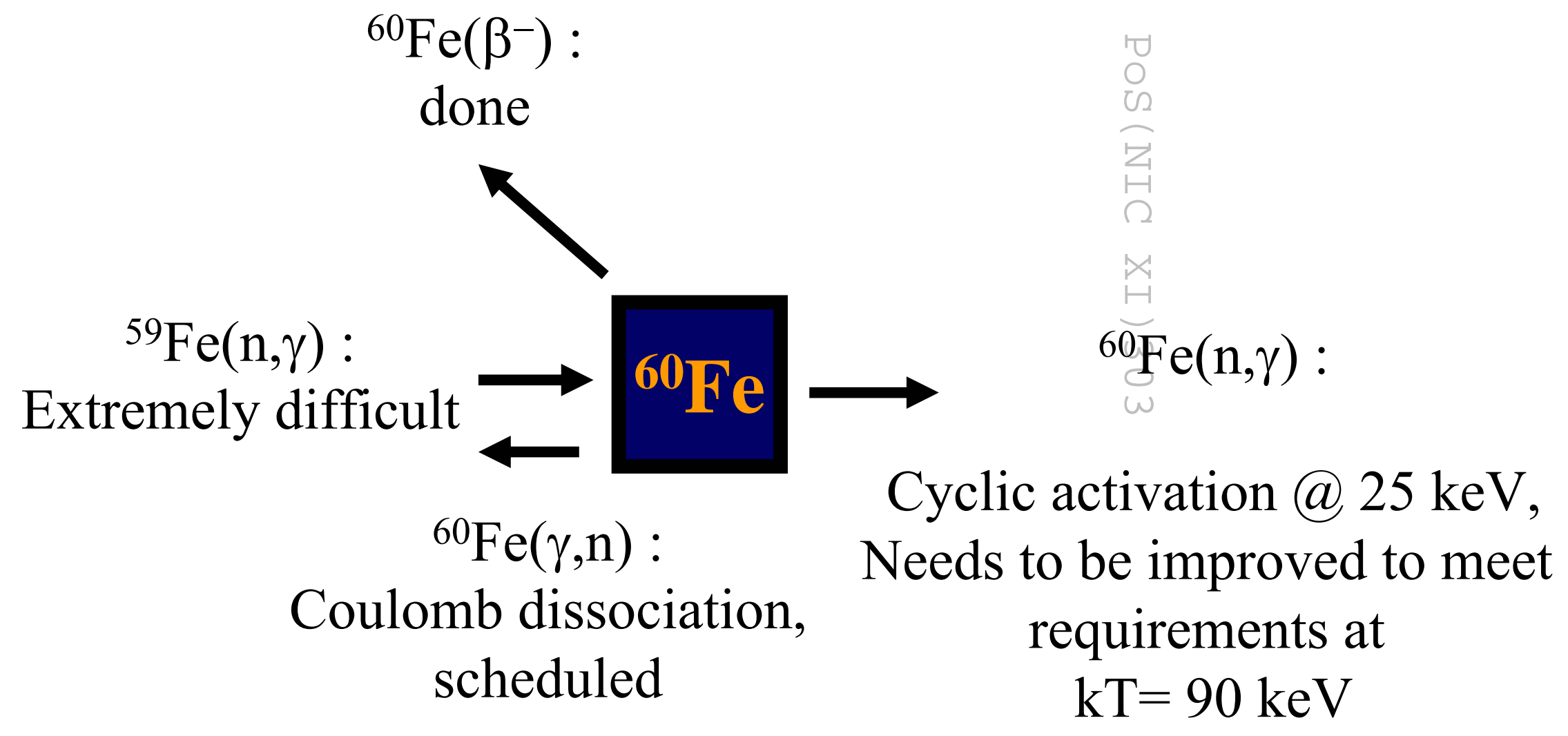




\section{Double neutron capture}

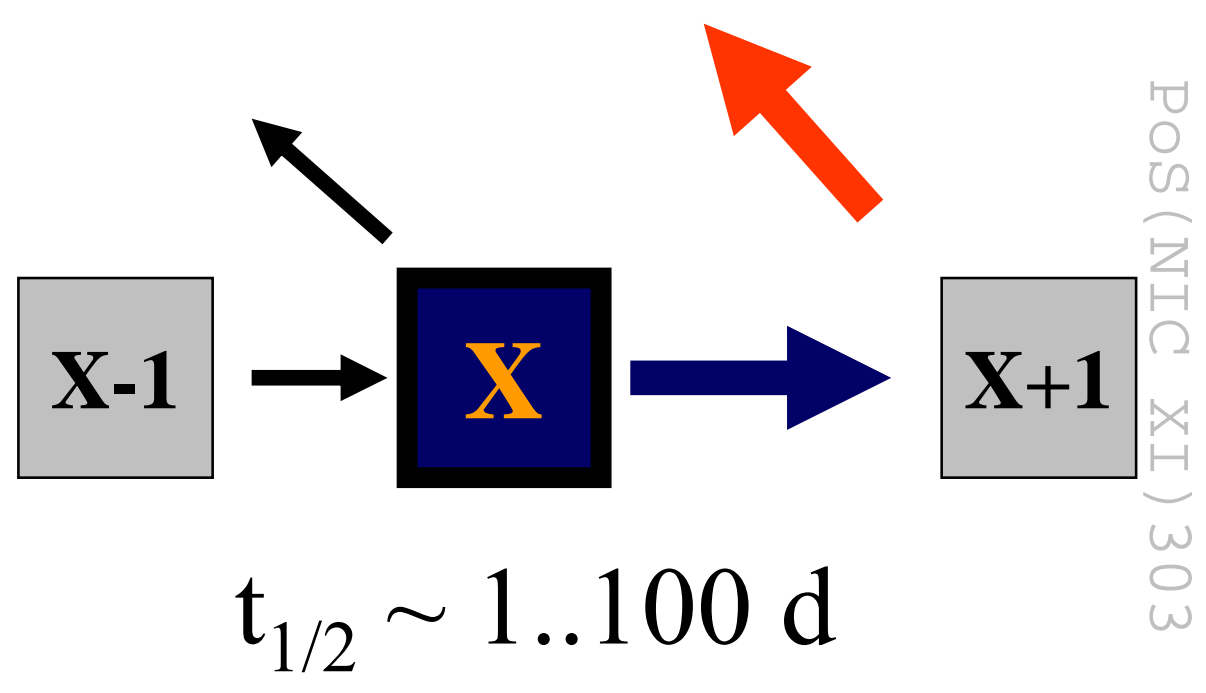

- produce the sample "on the fly"

- $10^{12} \mathrm{n} / \mathrm{s} / \mathrm{cm}^{2} @ 25 \mathrm{keV} \sim 510^{3} \mathrm{n} / \mathrm{cm}^{3}$ 


\section{${ }^{59} \mathrm{Fe}(\mathrm{n}, \gamma)$ at FRANZ $\left(\mathrm{t}_{1 / 2}=45 \mathrm{~d}\right)$}

- activate ${ }^{58} \mathrm{Fe}$, wait for $2^{\text {nd }}$ neutron capture

- measure ${ }^{60} \mathrm{Fe} / 58 \mathrm{Fe}$ ratio via AMS

$10^{12}$ neutrons $/ \mathrm{s} / \mathrm{cm}^{2}$

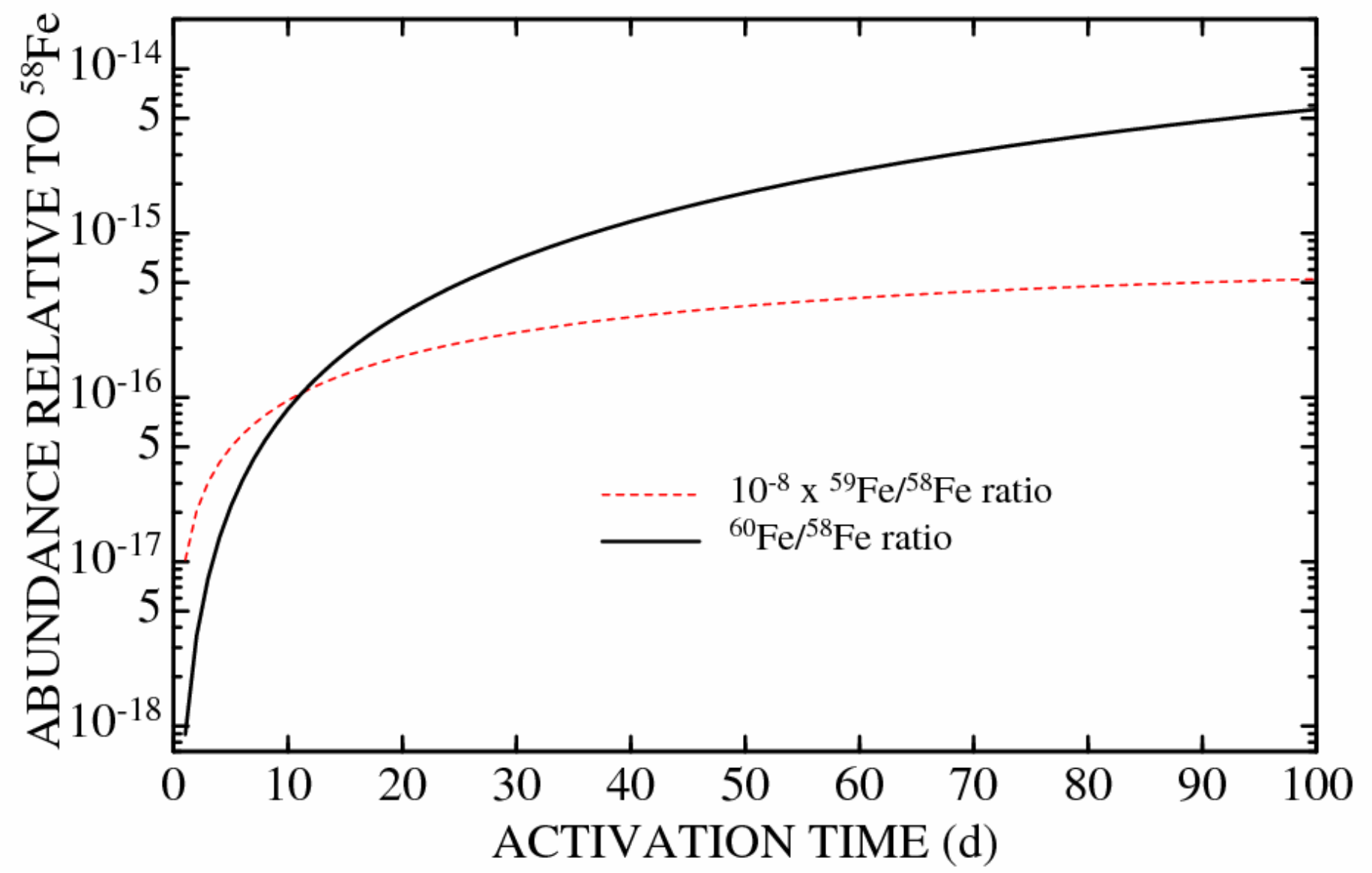

René Reifarth (GSI / U. Frankfurt) 


\section{Coulomb dissociation}

- Method for radioactive beams:

- Inverse kinematics

- "virtual photon field" as result of relativistic interaction with high-Z target (lead)

- Produce beam of radioactive ions

- In-beam experiment

- Detect ALL prompt products

- Gammas

- Ions 


\section{Experimental method}

Astrophysically relevant energy window: $E_{\mathrm{V}} \approx \mathrm{S}_{\mathrm{n}}+\mathrm{kT} / 2=8-12 \mathrm{MeV}$, width $\sim 1 \mathrm{MeV}$

Coulomb dissociation in inverse kinematics:

- Virtual photons produced by a high- $Z$ target $(\mathrm{Pb})$

- Projectile at $\sim 500 \mathrm{MeV} / \mathrm{u}$

- Large impact parameter $b$

- $\mathrm{E}_{\max }$ of the virtual photon spectrum $\sim 20 \mathrm{MeV}$

- $\quad$ and empty target measurements (to subtract

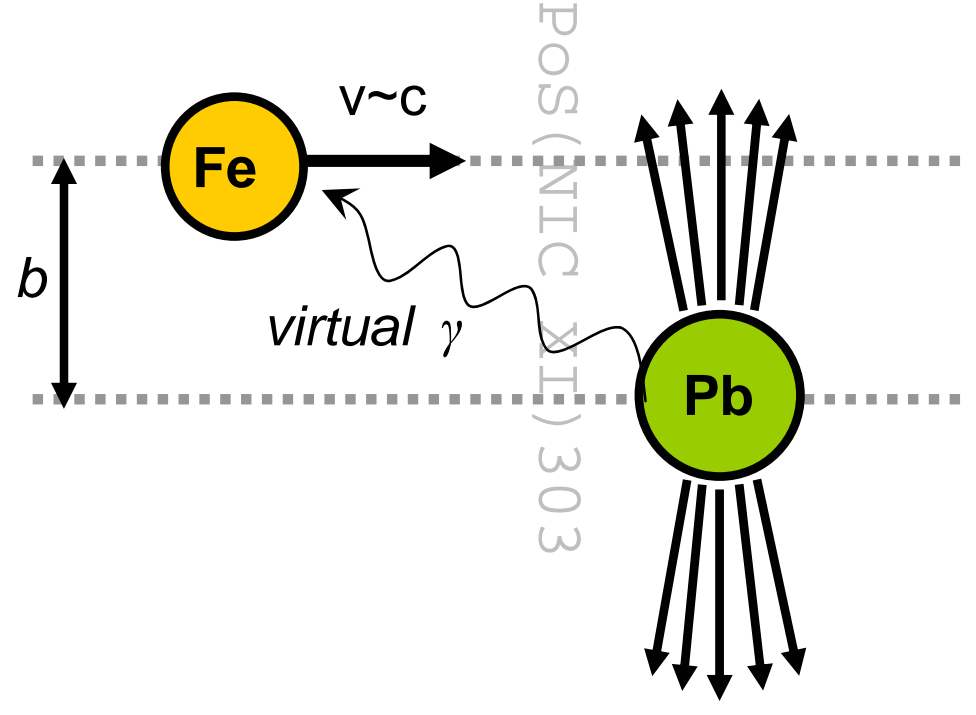
nuclear contribution and background) 


\section{Layout of the experimental facilities at GSI}

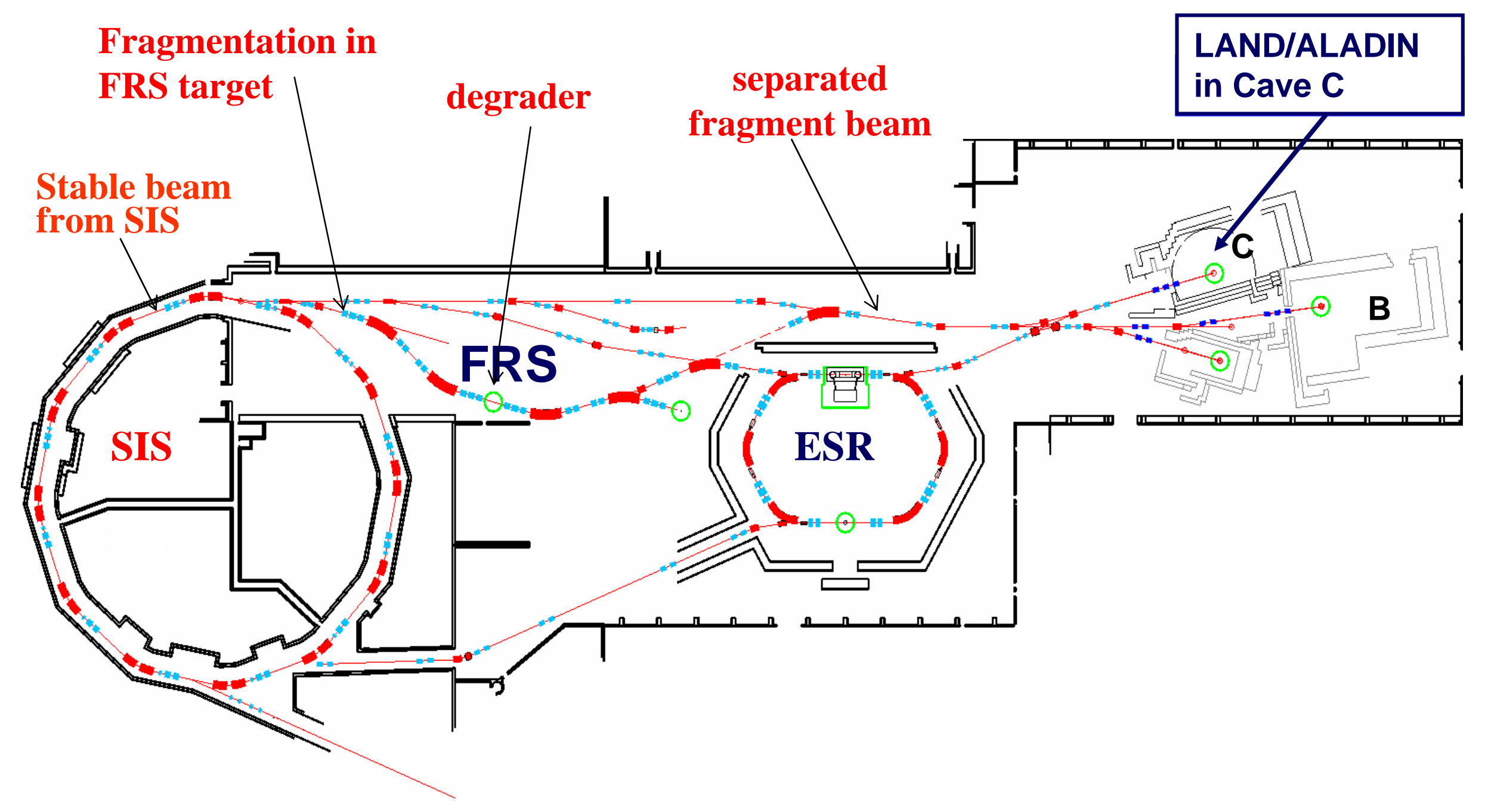

René Reifarth (GSI / U. Frankfurt) 


\section{$\mathbf{R}^{3} \mathbf{B}$ - Reactions with Relativistic Radioactive Beams}

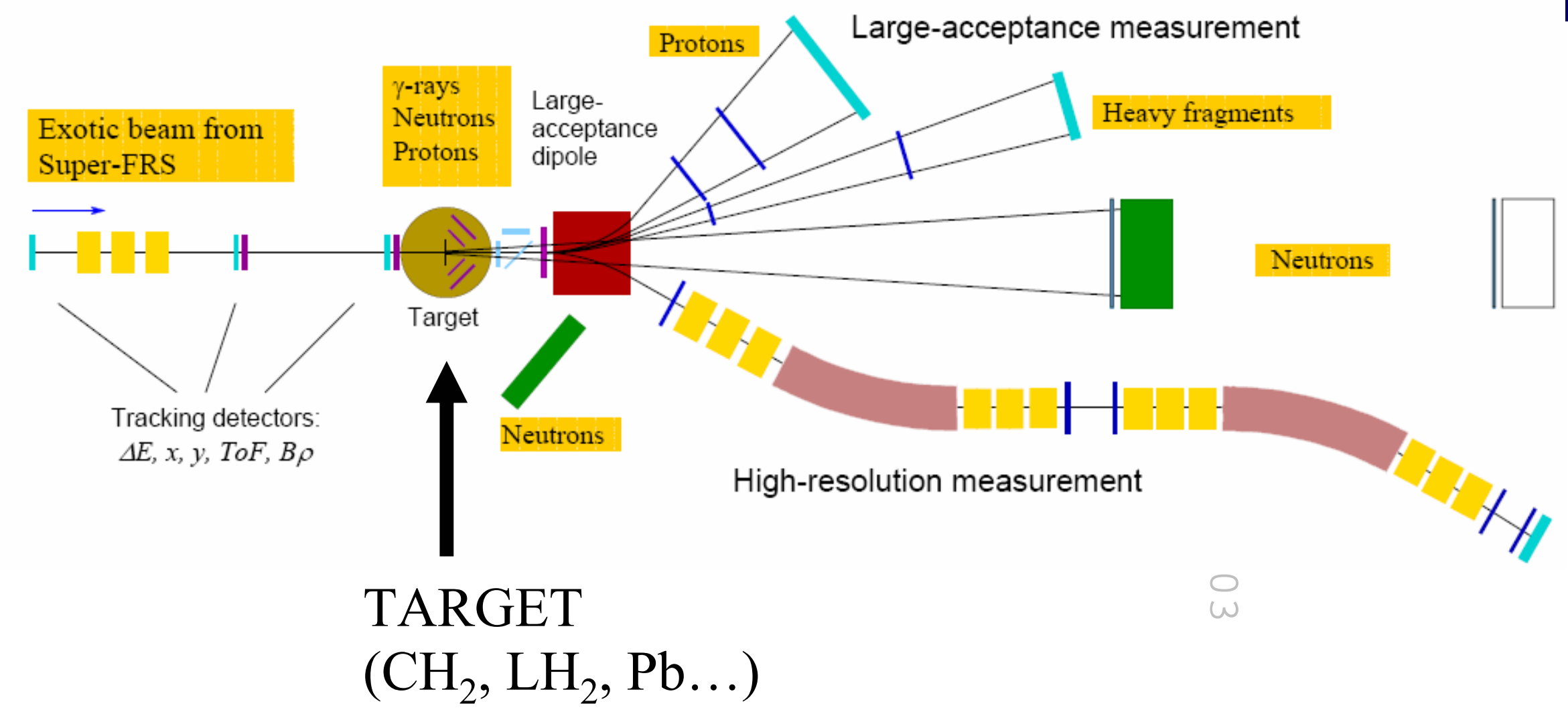

$\sim 100-\sim 1000 \mathrm{AMeV}$

From: $\mathbf{R}^{\mathbf{3}} \mathbf{B}$

Technical Report 


\section{Summary}

- $n$-induced reactions are important for nucleosynthesis beyond iron

- s-process can be used as a tool to constrain stellar parameters, if the corresponding reaction rates are known

- we are now close to measure n-induced cross section at stellar energies on radioactive nuclei on a routinely basis

- So far almost all measurements are done on stable nuclei 